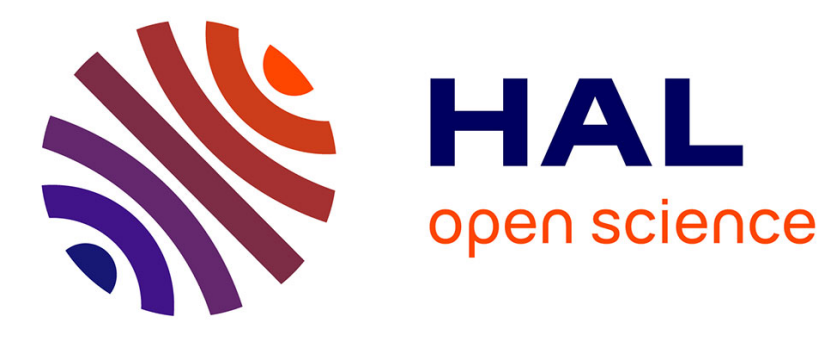

\title{
More Efficient Constructions for Inner-Product Encryption
}

Somindu C Ramanna

\section{To cite this version:}

Somindu C Ramanna. More Efficient Constructions for Inner-Product Encryption. Applied Cryptography and Network Security (ACNS 2016), Jun 2016, Guildford, United Kingdom. pp.231 - 248, 10.1007/978-3-319-39555-5_13. hal-01394288

\section{HAL Id: hal-01394288 \\ https://inria.hal.science/hal-01394288}

Submitted on 9 Nov 2016

HAL is a multi-disciplinary open access archive for the deposit and dissemination of scientific research documents, whether they are published or not. The documents may come from teaching and research institutions in France or abroad, or from public or private research centers.
L'archive ouverte pluridisciplinaire HAL, est destinée au dépôt et à la diffusion de documents scientifiques de niveau recherche, publiés ou non, émanant des établissements d'enseignement et de recherche français ou étrangers, des laboratoires publics ou privés. 


\title{
More Efficient Constructions for Inner-Product Encryption
}

\author{
Somindu C. Ramanna \\ Laboratoire LIP, ENS de Lyon, France \\ e-mail: somindu.ramanna@ens-lyon.fr
}

\begin{abstract}
We propose new constructions for inner product encryption - IPE $\mathcal{E}_{1}$ and $I P \mathcal{E}_{2}$, both secure under the eXternal Diffie-Hellman assumption (SXDH) in asymmetric pairing groups. The first scheme has constant-size ciphertexts whereas the second one is weakly attribute hiding. $I P E_{2}$ is derived from the identity-based encryption scheme of Jutla Roy (Asiacrypt 2013), that was extended from tag-based quasi-adaptive non-interactive zero-knowledge (QA-NIZK) proofs for linear subspaces of vector spaces over bilinear groups. The verifier common reference string (CRS) in these tag-based systems are split into two parts, that are combined during verification. We consider an alternate form of the tag-based QA-NIZK proof with a single verifier CRS that already includes a tag, different from the one defining the language. The verification succeeds as long as the two tags are unequal. Essentially, we embed a two-equation revocation mechanism in the verification. The new QA-NIZK proof system leads to $I \mathcal{P E}_{1}$, a constant-sized ciphertext IPE scheme with very short ciphertexts. Both the IPE schemes are obtained by applying the $n$ equation revocation technique of Attrapadung and Libert (PKC 2010) to the corresponding identity based encryption schemes and proved secure under SXDH assumption. As an application, we show how our schemes can be specialised to obtain the first fully secure identity-based broadcast encryption based on SXDH with a trade-off among the public parameters, ciphertext and key sizes, all of them being sub-linear in the maximum number of recipients of a broadcast.

Keywords: inner-product encryption, attribute-hiding, constant-size ciphertexts, quasi-adaptive non-interactive zero knowledge proofs.
\end{abstract}

\section{Introduction}

Inner product encryption (IPE) is a special form of the more general attributebased encryption (ABE), which provides fine-grained access control to encrypted data. In ABE, a ciphertext is encrypted to some attribute $\mathbf{x}$ and a secret key is associated to some attribute $\mathbf{y}$ such that decryption succeeds iff some relation $R$ on $\mathbf{x}, \mathbf{y}$ holds true i.e., $R(\mathbf{x}, \mathbf{y})=1$. The standard notion of security for $\mathrm{ABE}$ requires resistance to collusion attacks. More precisely, the privacy of a message encrypted to attribute $\mathbf{x}$ must not be compromised in the event of an attack by a group of users possessing secret keys for $\mathbf{y}_{1}, \mathbf{y}_{2}, \ldots, \mathbf{y}_{q}$ where $R\left(\mathbf{x}, \mathbf{y}_{i}\right)=0$ for all $i=1, \ldots, q$. Another useful security property, called weak 
attribute hiding, requires that given a ciphertext, the group of corrupt users unauthorised to decrypt the ciphertext, learn nothing about the attribute $\mathbf{x}$. In both cases, adaptive security allows users to be corrupted adaptively.

A simple form of ABE is identity-based encryption, where $\mathbf{x}$ and $\mathbf{y}$ represent identities and the relation $R$ tests equality of identities. IPE is a more complex form with $R$ testing orthogonality of $\mathbf{x}$ and $\mathbf{y}$ that are vectors in some inner product space. In other words, $R(\mathbf{x}, \mathbf{y})=1$ if $\langle\mathbf{x}, \mathbf{y}\rangle=0$ and 0 otherwise. Though they appear restricted, inner products cover a wide range of functionalities useful in practice including polynomial functions, boolean formulae evaluating conjunctive and disjunctive normal forms, and identity-based broadcast encryption and revocation.

Most efficient constructions of IPE are based on pairings. A pairing $e: \mathbb{G}_{1} \times$ $\mathbb{G}_{2} \rightarrow \mathbb{G}_{T}$ is a bilinear, non-degenerate and efficiently computable map defined over three groups $\mathbb{G}_{1}, \mathbb{G}_{2}, \mathbb{G}_{T}$ all having the same order. The common order of the groups may be composite or prime. Prime order pairings where $\mathbb{G}_{1} \neq \mathbb{G}_{2}$ are called asymmetric. The best choices for implementation are asymmetric pairings, particularly those with no efficiently computable isomorphisms between $\mathbb{G}_{1}$ and $\mathbb{G}_{2}$ (called Type-3 pairings), from a point of view of security as well as efficiency. A consequence of the absence of efficient isomorphisms makes the decisional Diffie-Hellman (DDH) problem hard in both groups $\mathbb{G}_{1}$ and $\mathbb{G}_{2}$, collectively called the symmetric eXternal decisional Diffie-Hellman (SXDH) problem. We mainly focus on security under this assumption.

A powerful technique to obtain adaptive security for attribute-based encryption schemes is the dual system methodology introduced by Waters [Wat09]. Important features of the underlying algebraic structure that facilitate a dual system proof are cancelling and parameter-hiding. These features are explicitly available in composite order pairing groups that are not really suitable for practical deployment. A number of works have investigated the possibilities of translating the properties of composite order pairings to the prime-order setting, mostly in the context of dual system hierarchical IBE and ABE. However, the constructions resulting from these translations are not necessarily optimised in terms of various system parameters (such as ciphertext/key size, time required for decryption and so on). In contrast, direct constructions in the prime-order setting circumventing the route via composite order pairings, holds more promise in this regard. We believe that IPE as a cryptographic primitive is significant enough to justify attempts for direct constructions.

The goal of this work is to obtain new direct Type-3 pairing-based constructions of IPE that are efficient, adaptively secure with a focus on achieving either of the following properties - attribute-hiding or compact ciphertexts - from the SXDH assumption.

Our Contributions. We propose two new IPE schemes based on prime-order pairings named $I \mathscr{P E} \mathcal{E}_{1}$ and $I P E_{2}$ - the former with constant-sized ciphertexts and the latter achieving weak attribute hiding, both secure under the SXDH assumption. The constructions are derived from quasi-adaptive non-interactive zero knowledge (QA-NIZK) proofs of Jutla and Roy [JR13] and an IBE proposed 
in the same work (denoted $\mathscr{I R}-I \mathcal{B E}$ in the rest of the paper). $I \mathcal{P E}_{2}$ is obtained from $\mathcal{I R}-I \mathcal{B E}$ by a novel application of the $n$-equation revocation technique of Attrapadung and Libert [AL10]. But a constant-size ciphertext IPE cannot be constructed in a similar way from $\mathscr{I R}-I \mathcal{B E}$. To get around this problem, we propose a small tweak to the Jutla-Roy QA-NIZK proofs that leads to an alternate form of $\mathcal{I R}-I \mathcal{B E}$ (named $\mathcal{I R}-I \mathcal{B E}-\mathcal{D}$ ). The $n$-equation revocation method is then combined with $\mathscr{I R}-I \mathcal{B E}-\mathcal{D}$ to construct $I \mathcal{P E} \mathcal{E}_{1}$. QA-NIZK proofs were only known to yield IBE [JR13], hierarchical IBE (HIBE) [RS14b] and identitybased broadcast encryption [RS14a] but the question of whether they are useful in constructing other forms of ABE remained open. Thus, we (partially) settle an open question posed in [CGW15].

Tables 1 and 2 compare our constructions to those recently proposed by Chen, Gay and Wee [CGW15]. The reason we do not include other previous constructions in the comparison is that the constructions in [CGW15] are the most efficient instantiations known so far and their constructions achieve security from the SXDH assumption. First, we define some abbreviations/notation we use in the comparison. \#pp, \#cpr and \#key denote the sizes of public parameters, ciphertexts and keys respectively. \#dec denotes the time required for decryption. $|X|$ denotes the size of representation of an element from $X$. [P], $\left[\mathrm{M}_{i}\right]$ (for $i=$ $1,2)$ and $[\mathrm{E}]$ respectively denote the time required for pairing operation, scalar multiplication in $\mathbb{G}_{i}$ (for $i=1,2$ ) and exponentiation in $\mathbb{G}_{T}$ respectively.

\begin{tabular}{l||c|c|c|c}
\hline Scheme & \#pp & \#cpr & \#key & \#dec \\
\hline \hline$[$ CGW15] & $(2 n+4)\left|\mathbb{G}_{1}\right|+\left|\mathbb{G}_{T}\right|$ & $4\left|\mathbb{G}_{1}\right|+\left|\mathbb{G}_{T}\right|$ & $(2 n+2)\left|\mathbb{G}_{2}\right|$ & $4[\mathrm{P}]+2 n\left[\mathrm{M}_{2}\right]$ \\
\hline$I P \mathcal{E}_{1}$ & $(n+3)\left|\mathbb{G}_{1}\right|+\left|\mathbb{G}_{T}\right|$ & $3\left|\mathbb{G}_{1}\right|+\left|\mathbb{Z}_{p}\right|+\left|\mathbb{G}_{T}\right|$ & $(2 n+1)\left|\mathbb{G}_{2}\right|+(n-1)\left|\mathbb{Z}_{p}\right|$ & $3[\mathrm{P}]+(2 n-2)\left[\mathrm{M}_{2}\right]+[\mathrm{E}]$ \\
\hline
\end{tabular}

Table 1. Constant-size ciphertext IPE.

\begin{tabular}{l||c|c|c|c}
\hline Scheme & \#pp & \#cpr & \#key & \#dec \\
\hline \hline [CGW15] & $(2 n+4)\left|\mathbb{G}_{1}\right|+\left|\mathbb{G}_{T}\right|$ & $(2 n+2)\left|\mathbb{G}_{1}\right|+\left|\mathbb{G}_{T}\right|$ & $4\left|\mathbb{G}_{2}\right|$ & $4[\mathrm{P}]+2 n\left[\mathrm{M}_{1}\right]$ \\
\hline$I P E_{2}$ & $(n+3)\left|\mathbb{G}_{1}\right|+\left|\mathbb{G}_{T}\right|$ & $(n+1)\left|\mathbb{G}_{1}\right|+(n-1)\left|\mathbb{Z}_{p}\right|+\left|\mathbb{G}_{T}\right|$ & $5\left|\mathbb{G}_{2}\right|$ & $3[\mathrm{P}]+(n+1)\left[\mathrm{M}_{1}\right]$ \\
\hline
\end{tabular}

Table 2. Attribute-hiding IPE.

Note that both our schemes are at least as efficient as the corresponding instantiations in [CGW15]. The public parameters and decryption time are better

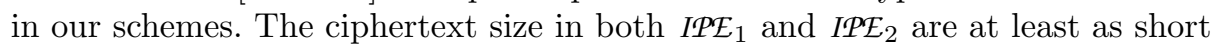
as those in [CGW15].

Quasi-Adaptive NIZK Proofs to IPE. Jutla and Roy [JR13] proposed constructions of quasi-adaptive non-interactive zero knowledge (QA-NIZK) proofs 
for linear equations over pairing groups that have a weaker soundness criterion called quasi-adaptive soundness. The difference with regular NIZKs is that the common reference string (CRS) is allowed to depend on the language. These are useful in constructing a number of primitives, such as signatures, CCA2secure public key encryption, commitment schemes and so on. From the signature scheme, they obtained an IBE using Naor's transform, which is the most efficient IBE known till date in terms of size of public parameters and ciphertexts achieving adaptive security under standard assumptions. Building upon this IBE, we obtain a weakly attribute hiding IPE scheme using the $n$-equation revocation method proposed in [AL10].

The NIZK construction that leads to the IBE is actually a split-CRS NIZK for tag-based languages, where the CRS for the verifier is split into two components. These two components are then combined using a public random tag ctag, which is also a parameter defining the language. We make a slight modification by combining the two components of the split-CRS with another tag ktag and only providing the combination as the CRS. This ensures that verification is successful unless the two tags are equal, thus making unconditional failure of verification a possibility. Nevertheless, the probability of failure is negligible and this small modification leads to an IBE scheme that has tags in both ciphertexts and keys. Decryption requires the two-equation revocation technique of Sahai and Waters [LSW08] as used in Waters' IBE [Wat09] and fails unconditionally with (negligible) probability equal to that of NIZK verification failure. The resulting IBE which we denote as $\mathcal{I R}-I \mathcal{B E}-\mathcal{D}$, allows extension to primitives that were not possible from $\mathcal{I R}-I \mathcal{B E}$, such as identity-based revocation schemes with small secret keys, constant-size ciphertext IBBE and so on. We present a construction of constant-size ciphertext IPE that can then be specialised to the afore-mentioned primitives. Unlike earlier constructions based on dual pairing vector spaces, specialising the IPE to specific cases actually leads to optimal constructions, i.e., these schemes are as efficient as direct constructions obtained from $\mathscr{I R}-I \mathcal{B E}-\mathcal{D}$.

The reason for first constructing an IBE is two-fold. Firstly, it provides better intuition and acts as a basis for moving to inner product functionality. Second and most importantly, we do not know a direct generic transformation from QA-NIZK proofs to IBE, let alone IPE. To this end, there has been some recent work [JR15] that defines the so-called dual system simulation sound QA-NIZK proofs that explain the $\mathcal{I R}-I \mathcal{B E}$ construction better in generic terms. It may be possible to explain our constructions too within this framework.

Application. As an application of IPE, we consider identity-based broadcast encryption (IBBE) wherein the goal is to securely broadcast an encrypted message to users associated with identities so that only a subset of privileged users can decrypt the message. Unlike the public key broadcast setting where the number of public keys varies polynomially with the security parameter, the number of valid identities in an IBBE are allowed to be exponential. Some direct constructions of adaptively secure constructions of IBBE schemes already exist in the literature [GW09,AL10,RS14a]. Most of these schemes require the number 
of privileged recipients for any broadcast to be bounded during setup (call this bound $n$ ). Previous schemes had either constant-sized ciphertexts or constantsized keys with at least one out of public parameters, ciphertext, key having size depending linearly on $n$.

We show how to construct an IBBE from $I P E_{1}$ that achieves parameters, ciphertexts and keys all having size sublinear in $n$ while maintaining security under static complexity assumptions. (Here, static means that the number of elements in instance is a constant). Due to lack of space, we present this discussion in Appendix C.

Related Work. There have been several constructions of attribute encryption schemes based on pairings [SW05, GPSW06, OSW07,BSW07, Wat11,LW12], some focussing only on inner product encryption [KSW08, OT09,OT10,AL10]. Latticebased constructions include ABE of [Boy13] for formulas and [GVW13, $\mathrm{GGH}^{+}$13] for circuits. We are mostly interested in constructions based on bilinear maps with prime order. Several approaches have been taken to constructing ABE schemes in the prime order pairing setting, most of them attempting to simulate properties of composite order pairings in suitably defined primeorder counterparts. A widely used technique is based on dual pairing vector spaces [OT08,OT09] which obtains all the nice theoretical properties but fails to preserve efficiency. The sparse DPVS technique introduced in [OT11] uses subgroups of sparse matrices (those mostly covered with zero entries) with the hope of improving efficiency. But the conversions are no longer generic and involve very complex security analysis. Another generic technique is that of dual system groups [CW13] that provides more efficient translations in the context of IBE. However, it does not extend to primitives that require anonymity or attribute-hiding. Two works [Wee14,Att14] present unifying frameworks for predicate encryption schemes fully secure within the dual system framework. These frameworks were defined in the composite order setting and later translated to prime-order groups [CGW15,Att15]. The translations to prime-order setting required additional restrictions on the structure of encodings that do not hold for most encodings proposed in [Wee14,Att14]. A recent work [AC16] provides a new instantiation of the encoding framework of [Att14] employing dual system groups ([CW13,CGW15]), thus implying constructions for predicate encryption in both composite-order and prime-order bilinear group settings. This work also considers a relaxation of the information theoretic security property used in [Att14,Att15] that allows covering a larger class of encodings. On the other hand, the efficiency tradeoffs for the constructions in [CGW15] and $[\mathrm{AC} 16]$ are directly related to the underlying dual system groups (DSG) instantiation. The DSG realisation in [CGW15] leads to very efficient constructions in the prime-order setting for several predicates. Apart from translations from composite-order groups, there have been attempts at direct constructions of certain simple primitives such as IBE and HIBE. The approach of [JR13] is via QANIZK proofs. This was later extended to HIBE in [RS14b] and IBBE [RS14a]. Another interesting approach was to construct $(\mathrm{H}) \mathrm{IBE}$ from message authentica- 
tion codes (which is a symmetric primitive), examined in [BKP14]. But it is not known whether or not the last method extends to attribute-based encryption.

\section{Preliminaries}

This section introduces some notation followed by a review of pairings and related hardness assumptions. Also provided are definitions related to innerproduct encryption.

\subsection{Notation}

The notation $x_{1}, \ldots, x_{k} \stackrel{\mathrm{R}}{\longleftarrow} \mathcal{X}$ indicates that elements $x_{1}, \ldots, x_{k}$ are sampled independently from the set $\mathcal{X}$ according to some distribution $\mathrm{R}$. We use $\mathrm{U}$ to denote the uniform distribution. For a (probabilistic) algorithm $\mathcal{A}, y \stackrel{\mathrm{R}}{\longleftarrow} \mathcal{A}(x)$ means that $y$ is chosen according to the output distribution of $\mathcal{A}$ on input $x$. $\mathcal{A}(x ; r)$ denotes that $\mathcal{A}$ is run on input $x$ with its internal random coins set to $r$. For two integers $a<b$, the notation $[a, b]$ represents the set $\{x \in \mathbb{Z}: a \leq x \leq b\}$. If $\mathbb{G}$ is a finite cyclic group, then $\mathbb{G}^{\times}$denotes the set of generators of $\mathbb{G}$.

We denote vectors in $\mathbb{Z}_{p}^{n}$ by bold upright characters (e.g. $\mathbf{x}$ ). Inner product of two $\mathbb{Z}_{p}^{n}$-vectors $\mathbf{x}=\left(x_{1}, \ldots, x_{n}\right)$ and $\mathbf{y}=\left(y_{1}, \ldots, y_{n}\right)$ is given by $\langle\mathbf{x}, \mathbf{y}\rangle=$ $\sum_{i=1}^{n} x_{i} y_{i}$.

\subsection{Asymmetric Pairings and Hardness Assumptions}

A bilinear pairing ensemble is a 7 -tuple $\mathcal{G}=\left(p, \mathbb{G}_{1}, \mathbb{G}_{2}, \mathbb{G}_{T}, e, P_{1}, P_{2}\right)$ where $\mathbb{G}_{1}=\left\langle P_{1}\right\rangle, \mathbb{G}_{2}=\left\langle P_{2}\right\rangle$ are written additively and $\mathbb{G}_{T}$ is a multiplicatively written group, all having the same order $p$ and $e: \mathbb{G}_{1} \times \mathbb{G}_{2} \rightarrow \mathbb{G}_{T}$ (the pairing) is a bilinear, non-degenerate and efficiently computable map. In a Type-3 pairing, $\mathbb{G}_{1} \neq \mathbb{G}_{2}$ and no efficiently computable isomorphisms between $\mathbb{G}_{1}$ and $\mathbb{G}_{2}$ are known. The constructions we provide are based on such pairings.

The assumptions based on which the security of our constructions is proven are the decision Diffie-Hellman (DDH) assumptions in groups $\mathbb{G}_{1}$ and $\mathbb{G}_{2}$, called DDH1 and DDH2 respectively. Below, we describe these two assumptions. Technically speaking, the two assumptions are not in the standard form but can be shown to be equivalent. The reason we use the alternate forms is that they suit the requirements of our reductions and also to be in sync with the notation in [JR13].

Let $\mathcal{G}=\left(p, \mathbb{G}_{1}, \mathbb{G}_{2}, \mathbb{G}_{T}, e, P_{1}, P_{2}\right)$ be an asymmetric pairing ensemble and $\mathscr{A}$, a probabilistic polynomial time (PPT) algorithm $\mathscr{A}$ that outputs 0 or 1 .

Assumption DDH1. Define a distribution $\mathcal{D}$ as follows: $P_{1} \stackrel{\mathrm{U}}{\longleftarrow} \mathbb{G}_{1}^{\times} ; b, s \stackrel{\mathrm{U}}{\longleftarrow}$ $\mathbb{Z}_{p}, \mu \stackrel{\mathrm{U}}{\longleftarrow} \mathbb{Z}_{p} ; \mathcal{D}=\left(\mathcal{G}, P_{1}, b P_{1}, b s P_{1}\right)$. The advantage of $\mathscr{A}$ in solving the DDH1 problem is given by

$$
\operatorname{Adv}_{\mathcal{G}}^{\mathrm{DDH} 1}(\mathscr{A})=\left|\operatorname{Pr}\left[\mathscr{A}\left(\mathcal{D}, s P_{1}\right)=1\right]-\operatorname{Pr}\left[\mathscr{A}\left(\mathcal{D},(s+\mu) P_{1}\right)=1\right]\right| .
$$


Essentially, $\mathscr{A}$ has to decide whether $\mu=0$ or $\mu \in_{\mathrm{U}} \mathbb{Z}_{p}$ given $\left(\mathcal{D},(s+\mu) P_{1}\right)$. The $(\varepsilon, t)$-DDH1 assumption holds in $\mathcal{G}$ if for any adversary $\mathscr{A}$ running in time at most $t, \operatorname{Adv}_{\mathcal{G}}^{\mathrm{DDH} 1}(\mathscr{A}) \leq \varepsilon$.

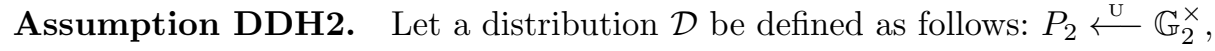
$r, c \stackrel{\mathrm{U}}{\longleftarrow} \mathbb{Z}_{p}, \gamma \stackrel{\mathrm{U}}{\longleftarrow} \mathbb{Z}_{p}$

$$
\mathcal{D}=\left(\mathcal{G}, P_{2}, r P_{2}, c P_{2}\right) .
$$

$\mathscr{A}$ 's advantage in solving the DDH2 problem is given by

$$
\operatorname{Adv}_{\mathcal{G}}^{\mathrm{DDH} 2}(\mathscr{A})=\left|\operatorname{Pr}\left[\mathscr{A}\left(\mathcal{D}, r c P_{2}\right)=1\right]-\operatorname{Pr}\left[\mathscr{A}\left(\mathcal{D},(r c+\gamma) P_{2}\right)=1\right]\right| .
$$

The $(\varepsilon, t)$-DDH2 assumption is that, for any $t$-time algorithm $\mathscr{A}, \operatorname{Adv}_{\mathcal{G}}^{\mathrm{DDH} 2}(\mathscr{A}) \leq$ $\varepsilon$.

\subsection{Inner Product Encryption (IPE)}

Definition 1 (IPE). Let $V$ denote a vector space of dimension $n$ over a field $\mathbb{F}$ and $\mathcal{M}$ denote the message space. An IPE scheme for inner products over $V$, is defined by four probabilistic algorithms - Setup, Encrypt, KeyGen and Decrypt.

Setup $(\kappa, n)$ Takes as input a security parameter $\kappa$ and the dimension of $V$. It outputs the public parameters $\mathcal{P} \mathcal{P}$ and the master secret $\mathcal{M S K}$.

KeyGen $(\mathcal{M S K}, \mathbf{y})$ On input a vector $\mathbf{y} \in V$ and the master secret $\mathcal{M S K}$; this algorithm outputs a secret key $\mathcal{S} \mathcal{K}_{\mathbf{y}}$ for $\mathbf{y}$.

$\operatorname{Encrypt}(\mathcal{P} \mathcal{P}, m, \mathbf{x})$ Takes as input a message $m$ and an attribute vector $\mathbf{x} \in V$ and outputs a ciphertext $\mathcal{C}$.

$\operatorname{Decrypt}\left(\mathcal{P} \mathcal{P}, \mathcal{C}, \mathcal{S} \mathcal{K}_{\mathbf{y}}\right)$ If $\langle\mathbf{x}, \mathbf{y}\rangle=0$, this algorithm returns the message $m$ and $\perp$ otherwise.

Correctness. The IPE scheme is said to satisfy the correctness condition if for all vectors $\mathbf{x}, \mathbf{y} \in V$ with $\langle\mathbf{x}, \mathbf{y}\rangle=0$ and for all $m \in \mathcal{M}$, if $(\mathcal{P P}, \mathcal{M S K}) \stackrel{\mathrm{R}}{\longleftarrow}$ $\operatorname{Setup}(\kappa, n), \mathcal{S} \mathcal{K}_{\mathbf{y}} \stackrel{\mathrm{R}}{\longleftarrow} \operatorname{KeyGen}(\mathcal{M S K}, \mathbf{y}), \mathcal{C} \stackrel{\mathrm{R}}{\longleftarrow} \operatorname{Encrypt}(\mathcal{P} \mathcal{P}, m, \mathbf{x})$, then $\operatorname{Pr}[m=$ $\left.\operatorname{Decrypt}\left(\mathcal{P} \mathcal{P}, \mathcal{C}, \mathcal{S} \mathcal{K}_{\mathbf{y}}\right)\right]=1$.

Definition 2 (Security). The security definition for inner product encryption scheme that we consider is weak attribute hiding and adaptive security against chosen plaintext attacks. It is formalised in terms of the following game ind-wah-cpa between an adversary $\mathscr{A}$ and a challenger.

Setup: The challenger runs the Setup algorithm of the IPE and gives the public parameters to $\mathscr{A}$.

Key Extraction Phase 1: $\mathscr{A}$ makes a number of key extraction queries adaptively. For a query on a vector $\mathbf{y}$, the challenger responds with a key $\mathcal{S} \mathcal{K}_{\mathbf{y}}$.

Challenge: $\mathscr{A}$ provides two pairs of messages and attribute vectors $m_{0}, \widehat{\mathbf{x}}_{0}$ and $m_{1}, \widehat{\mathbf{x}}_{1}$ with the restriction that if $\mathbf{y}$ is queried in the key extraction phase 1 , then $\left\langle\widehat{\mathbf{x}}_{0}, \mathbf{y}\right\rangle \neq 0$ and $\left\langle\widehat{\mathbf{x}}_{1}, \mathbf{y}\right\rangle \neq 0$. The challenger chooses a bit $\beta$ uniformly at random from $\{0,1\}$, encrypts $m_{\beta}$ to $\widehat{\mathbf{x}}_{\beta}$ and returns the resulting ciphertext $\widehat{\mathcal{C}}$ to $\mathscr{A}$. 
Key Extraction Phase 2: $\mathscr{A}$ makes more key extraction queries with the restriction that it cannot query a key for any vector $\mathbf{y}$ with $\left\langle\widehat{\mathbf{x}}_{0}, \mathbf{y}\right\rangle=0$ or $\left\langle\widehat{\mathbf{x}}_{1}, \mathbf{y}\right\rangle=0$.

Guess: $\mathscr{A}$ outputs a bit $\beta^{\prime}$.

If $\beta=\beta^{\prime}$, then $\mathscr{A}$ wins the game. The advantage of $\mathscr{A}$ in winning the ind-wah-cpa is given by

$$
\operatorname{Adv}_{\text {IPE }}^{\text {ind-wah-cpa }}(\mathscr{A})=\left|\operatorname{Pr}\left[\beta=\beta^{\prime}\right]-\frac{1}{2}\right| .
$$

The IPE scheme is said to be $(\varepsilon, t, q)$-IND-WAH-CPA secure if every t-time adversary making at most $q$ key extraction queries has $\operatorname{Adv}_{I P E}^{\text {ind-wah-cpa }}(\mathscr{A}) \leq \varepsilon$.

We also consider a slightly weaker form of adaptive security denoted IND-CPA-security where attribute hiding property is not achieved. In the corresponding security game, denoted ind-cpa, $\widehat{\mathbf{x}}_{1}=\widehat{\mathbf{x}}_{2}$ that is, there is only one challenge attribute vector $\widehat{\mathbf{x}}$.

\section{Variant of Jutla-Roy Split-CRS NIZK Proof and IBE}

In this section, we suggest a small modification to QA-NIZK proofs of Jutla and Roy [JR13] and describe an IBE derived from it. We denote the IBE as IR $I \mathcal{B E}-\mathcal{D}$, the ' $\mathrm{d}$ ' signifying a sort of 'dual' of the original scheme. IR $-I \mathcal{B E}-\mathcal{D}$ forms the basis of our IPE construction with short ciphertexts. Since the QANIZK construction only points a way to the IBE construction, we provide an informal description of the modification required without delving into details of the construction or proof. For definitions and more details related to QA-NIZK proofs we refer to [JR13].

We are mainly interested in NIZK proofs for languages that are linear subspaces of vectors of $\mathbb{G}_{2}$-elements. [JR13] actually considers vectors over $\mathbb{G}_{1}$. Since $\mathbb{G}_{1}$ has shorter representation compared to $\mathbb{G}_{2}$, we prefer the ciphertext components to live in $\mathbb{G}_{1}$ and hence reverse the roles of $\mathbb{G}_{1}$ and $\mathbb{G}_{2}$ in our presentation. A linear subspace language is parameterised by an $t \times m$ matrix $\mathbf{A}$ of $\mathbb{G}_{2}$-elements and defined as

$$
L_{\mathbf{A}}=\left\{\mathbf{x}^{T} \mathbf{A} \mid \mathbf{x} \in \mathbb{Z}_{p}^{t}\right\}
$$

A NIZK proof system for this language is a collection of 4 algorithms $\left(\mathrm{K}_{0}, \mathrm{~K}_{1}, \mathrm{P}, \mathrm{V}\right)$ where $\mathrm{K}_{0}$ generates the common parameters (group descriptions for a pairing), $\mathrm{K}_{1}$ generates $\mathrm{CRS}_{p}$ and $\mathrm{CRS}_{v}$, the prover and verifier CRS's respectively, $\mathrm{P}$ generates a proof given a witness $\mathbf{x}$ for a candidate $\vec{Q} \in L_{\mathbf{A}}$ and $\mathrm{V}$ verifies that the proof is valid. Quasi-adaptiveness refers to the CRS being allowed to depend on the parameter, ( $\mathbf{A}$ in the above case). Three notions completeness, soundness and zero-knowledge - formalise the security requirements of a NIZK proof system. [JR13] starts with an efficient construction for this language and then extends it to what they call the split-CRS QA-NIZK system. The languages supported by such systems are characterised as

$$
L_{\mathbf{A}, \vec{A}_{1}, \vec{A}_{2}}=\left\{\mathbf{x}^{T} \cdot\left[\mathbf{A} \mid \vec{A}_{1}+\operatorname{ctag} \cdot \vec{A}_{2}\right] \mid \mathbf{x} \in \mathbb{Z}_{p}^{t}, \operatorname{ctag} \in \mathbb{Z}_{p}\right\},
$$


with $\mathbf{A} \in \mathbb{G}_{2}^{t \times m}, \overrightarrow{A_{1}}, \overrightarrow{A_{2}} \in \mathbb{G}_{2}^{t}$ are parameters defining the language. Writing $\mathbf{A}$ as $\left[\mathbf{A}_{l} \mid \mathbf{A}_{r}\right]$ with $\mathbf{A}_{l} \in \mathbb{G}_{2}^{t \times t}$ and $\mathbf{A}_{r} \in \mathbb{G}_{2}^{(m-t) \times t}$ and assuming that the number $(m-t)$ of equations in excess of the number of unknowns can be verified by just making additional randomised copies of the CRS [JR13], we only consider $\mathbf{A}_{l}$ in our descriptions. The algorithms of the split-CRS NIZK system are described below.

$\mathrm{K}_{0}$ : Generates the bilinear pairing parameters $\mathcal{G}=\left(p, \mathbb{G}_{1}, \mathbb{G}_{2}, \mathbb{G}_{T}, e, P_{1}, P_{2}\right)$.

$\mathrm{K}_{1}$ : Generates CRS as

$$
\begin{array}{ccc}
\mathrm{CRS}_{p, 0}=\left[\mathbf{A}_{l} \mid \vec{A}_{1}\right]\left[\begin{array}{c}
\mathbf{u}_{1} \\
b^{-1}
\end{array}\right] & \mathrm{CRS}_{p, 1}=\left[\mathbf{A}_{l} \mid \vec{A}_{2}\right]\left[\begin{array}{c}
\mathbf{u}_{2} \\
b^{-1}
\end{array}\right] \\
\mathrm{CRS}_{v, 0}=\left[\begin{array}{c}
b \mathbf{u}_{1} \\
1 \\
-b
\end{array}\right] P_{1} & \mathrm{CRS}_{v, 1}=\left[\begin{array}{c}
b \mathbf{u}_{2} \\
0 \\
0
\end{array}\right] P_{1},
\end{array}
$$

where $\mathbf{u}_{1}, \mathbf{u}_{2} \stackrel{\mathrm{U}}{\longleftarrow} \mathbb{Z}_{p}^{t}$ and $b \stackrel{\mathrm{U}}{\longleftarrow} \mathbb{Z}_{p}^{\times}$. Note that $\mathrm{CRS}_{v, 0}, \mathrm{CRS}_{v, 1} \in \mathbb{G}_{1}^{t+2}$.

$\mathrm{P}$ : Suppose the candidate is $\vec{Q}=\mathbf{x}^{T} \cdot\left[\mathbf{A} \mid \vec{A}_{1}+\operatorname{ctag} \cdot \vec{A}_{2}\right]$. The proof is given by

$$
\vec{R}=\mathrm{x}^{T}\left(\mathrm{CRS}_{p, 0}+\mathrm{ctag} \cdot \mathrm{CRS}_{p, 1}\right) .
$$

V: Given a proof $\vec{R}$ for a candidate $\vec{Q}$, the verifier checks whether

$$
e\left([\vec{R} \mid \vec{Q}], \mathrm{CRS}_{v, 0}+\operatorname{ctag} \cdot \mathrm{CRS}_{v, 1}\right)
$$

equals $1_{T}$, the identity of $\mathbb{G}_{T}$ or not indicating validity of the proof or otherwise, respectively. Here the pairing function $e$ evaluated on vectors is nothing but the product of the component-wise evaluations.

Our modification. We are now ready to propose our tweak to this splitCRS NIZK system. Instead of combining the verifier CRS's during verification, consider providing only one verifier CRS defined as

$$
\mathrm{CRS}_{v}=\mathrm{CRS}_{v, 0}+\operatorname{ktag} \mathrm{CRS}_{v, 1}
$$

where ktag $\stackrel{U}{\longleftarrow} \mathbb{Z}_{p}$ is chosen in $\mathrm{K}_{1}$. Verification is now done by testing whether

$$
e\left([\vec{R} \mid \vec{Q}], \mathrm{CRS}_{v}\right)^{\frac{1}{(\mathrm{ctag}-k \mathrm{ktg})}}
$$

is $1_{T}$ only if ctag $\neq \mathrm{ktag}$. Verification fails unconditionally if the two tags are equal. The modification weakens the quasi-adaptive soundness criterion since there is a probability that the verification algorithm fails. However, we make this modification only to make a transition to attribute-based encryption. Whether this NIZK system is actually useful for other purposes is beyond the scope of this work. 
IBE. We now present the identity-based encryption scheme obtained from the above mentioned NIZK system.

Setup $(\kappa)$ : Let $\mathcal{G}=\left(p, \mathbb{G}_{1}, \mathbb{G}_{2}, \mathbb{G}_{T}, e, F_{1}, F_{2}\right)$ be a Type-3 pairing ensemble generated based on the security parameter $\kappa$. Choose $P_{1} \stackrel{U}{\longleftarrow} \mathbb{G}_{1}^{\times}, P_{2} \stackrel{U}{\longleftarrow} \mathbb{G}_{2}^{\times}$, $b \stackrel{\mathrm{U}}{\longleftarrow} \mathbb{Z}_{p}^{\times}, \alpha_{1}, \alpha_{2}, u_{1}, u_{2}, v_{1}, v_{2}, w_{1}, w_{2} \stackrel{\mathrm{U}}{\longleftarrow} \mathbb{Z}_{p}$ and set $U_{1}=\left(u_{1}+b u_{2}\right) P_{1}, V_{1}=$ $\left(v_{1}+b v_{2}\right) P_{1}, W_{1}=\left(w_{1}+b w_{2}\right) P_{1}, g_{T}=e\left(P_{1}, P_{2}\right)^{\alpha_{1}+b \alpha_{2}}$. The parameters are given by

$$
\begin{aligned}
& \mathcal{P P}:\left(P_{1}, b P_{1}, U_{1}, V_{1}, W_{1}, g_{T}\right) \\
& \mathcal{M S K}:\left(P_{2}, \alpha_{1}, \alpha_{2}, u_{1}, u_{2}, v_{1}, v_{2}, w_{1}, w_{2}\right)
\end{aligned}
$$

$\operatorname{Encrypt}(\mathcal{P} \mathcal{P}, m$, id $):$ The ciphertext is given by $\mathcal{C}=\left(C_{0}, C_{1}, C_{2}, C_{3}\right.$, ctag $)$ where

$$
\begin{aligned}
& \text { ctag, } s \stackrel{\mathrm{U}}{\longleftarrow} \mathbb{Z}_{p}, \\
& C_{0}=m \cdot\left(g_{T}\right)^{s}, \\
& C_{1}=s P_{1}, C_{2}=s b P_{1}, C_{3}=s\left(U_{1}+\mathrm{id} V_{1}+\operatorname{ctag} W_{1}\right) .
\end{aligned}
$$

$\operatorname{KeyGen}(\mathcal{M S K}$, id $)$ Compute the secret key $\mathcal{S} \mathcal{K}_{\text {id }}=\left(K_{1}, K_{2}, K_{3}, K_{4}, K_{5}\right.$, ktag $)$ as follows.

$$
\begin{gathered}
r, \operatorname{ktag} \stackrel{\mathrm{U}}{\overleftarrow{Z}} \mathbb{Z}_{p}, \\
K_{1}=r P_{2}, K_{2}=\left(\alpha_{1}+r w_{1}\right) P_{2}, K_{3}=\left(\alpha_{2}+r w_{2}\right) P_{2} \\
K_{4}=r\left(u_{1}+\mathrm{id} v_{1}+\operatorname{ktag} w_{1}\right) P_{2}, K_{5}=r\left(u_{2}+\mathrm{id} v_{2}+\operatorname{ktag} w_{2}\right) P_{2} . \\
\operatorname{Decrypt}\left(\mathcal{C}, \mathcal{S} \mathcal{K}_{\text {id }}\right): \text { If ctag }=\mathrm{ktag}, \text { return } \perp . \text { Otherwise compute }
\end{gathered}
$$

$$
A=\left(\frac{e\left(C_{3}, K_{1}\right)}{e\left(C_{1}, K_{4}\right) e\left(C_{2}, K_{5}\right)}\right)^{\frac{1}{\operatorname{ctag}-k \operatorname{tag}}}
$$

and recover the message as

$$
m=\frac{C_{0} \cdot A}{e\left(C_{1}, K_{2}\right) e\left(C_{2}, K_{3}\right)} .
$$

The message $m$ can be recovered in a single step involving 3 pairing operations.

Decryption involves the two-equation revocation technique of Sahai and Waters [LSW08] that was also used in Waters IBE [Wat09]. The scheme is adaptively secure under the SXDH assumption. Since $\mathcal{I R}-I \mathcal{B E}-\mathcal{D}$ is a special case of $I \mathcal{P E}_{1}$, its security is implied by that of $I \mathcal{P E} \mathcal{E}_{1}$. Hence we omit the proof.

\section{IPE with Short Ciphertexts}

In this section, we define our first IPE construction IPE ${ }_{1}$ with constant-size ciphertexts and show that it is adaptively secure. As mentioned earlier, we use the $n$-equation revocation technique of Attrapadung and Libert [AL10] to extend IR $I \mathcal{B E}-\mathcal{D}$ to support inner product encryption. Below is the description of the

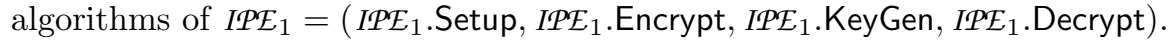


$I P E_{1}$.Setup $(\kappa, n)$ : Generate a Type-3 pairing $\mathcal{G}=\left(p, \mathbb{G}_{1}, \mathbb{G}_{2}, \mathbb{G}_{T}, e, F_{1}, F_{2}\right)$ based on the security parameter $\kappa$. Choose $P_{1} \longleftarrow \mathbb{G}_{1}^{\times}, P_{2} \longleftarrow \mathbb{G}_{2}^{\times}, b \stackrel{\mathrm{U}}{\longleftarrow} \mathbb{Z}_{p}^{\times}$, $\alpha_{1}, \alpha_{2}, w_{1}, w_{2} \stackrel{\mathrm{U}}{\longleftarrow} \mathbb{Z}_{p}, \mathbf{u}_{1}=\left(u_{1,1}, \ldots, u_{1, n}\right), \mathbf{u}_{2}=\left(u_{2,1}, \ldots, u_{2, n}\right) \stackrel{\mathrm{U}}{\longleftarrow} \mathbb{Z}_{p}^{n}$ and set $\mathbf{u}=\left(\mathbf{u}_{1}+b \mathbf{u}_{2}\right) P_{1}, w=\left(w_{1}+b w_{2}\right), g_{T}=e\left(P_{1}, P_{2}\right)^{\alpha_{1}+b \alpha_{2}}$. The parameters are given by

$\mathcal{P P}:\left(P_{1}, b P_{1}, \mathbf{u} P_{1}, w P_{1}, g_{T}\right)$

$\mathcal{M S K}:\left(P_{2}, \alpha_{1}, \alpha_{2}, \mathbf{u}_{1}, \mathbf{u}_{2}, w_{1}, w_{2}\right)$

$I \mathcal{P E}_{1}$. Encrypt $\left(\mathcal{P P}, m, \mathbf{x}=\left(x_{1}, \ldots, x_{n}\right)\right)$ : Components of the ciphertext are computed as follows.

$$
\begin{aligned}
& \text { ctag, } s \stackrel{\mathrm{U}}{\longleftarrow} \mathbb{Z}_{p}, \\
& C_{0}=m \cdot\left(g_{T}\right)^{s} \text {, } \\
& C_{1}=s P_{1}, C_{2}=s b P_{1}, C_{3}=s(\langle\mathbf{x}, \mathbf{u}\rangle+\operatorname{ctag} \cdot w) P_{1} .
\end{aligned}
$$

Note that $C_{3}$ can be computed from $\mathbf{u} P_{1}, w P_{1}$ and ctag using $n+1$ scalar multiplications. The ciphertext is given by $\mathcal{C}=\left(\mathbf{x}, C_{0}, C_{1}, C_{2}, C_{3}\right.$, ctag $)$.

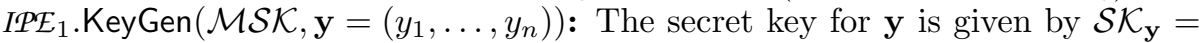
$\left(K_{1}, K_{2}, K_{3},\left(K_{4, i}, K_{5, i}, \operatorname{ktag}_{i}\right)_{i=2}^{n}\right)$ where

$$
\begin{aligned}
& r,\left(\operatorname{ktag}_{i}\right)_{i=2}^{n} \stackrel{\mathrm{U}}{\longleftarrow} \mathbb{Z}_{p}, \\
& K_{1}=r P_{2}, K_{2}=\left(\alpha_{1}+r w_{1}\right) P_{2}, K_{3}=\left(\alpha_{2}+r w_{2}\right) P_{2} \\
& \text { For } i=2, \ldots, n \text {, } \\
& K_{4, i}=r\left(-u_{1,1} \frac{y_{i}}{y_{1}}+u_{1, i}+\operatorname{ktag}_{i} w_{1}\right) P_{2}, K_{5, i}=r\left(-u_{2,1} \frac{y_{i}}{y_{1}}+u_{2, i}+\operatorname{ktag}_{i} w_{2}\right) P_{2} .
\end{aligned}
$$

$I \mathcal{P E}_{1}$. Decrypt $\left(\mathcal{C}, \mathcal{S} \mathcal{K}_{\mathbf{y}}\right):$ Compute ktag $=\sum_{i=2}^{n} x_{i} \mathrm{ktag}_{i}$. If ctag $=$ ktag, return $\perp$. Otherwise let

$$
A=\left(e\left(C_{3}, K_{1}\right) e\left(C_{1}, \sum_{i=2}^{n} x_{i} K_{4, i}\right)^{-1} e\left(C_{2}, \sum_{i=2}^{n} x_{i} K_{5}\right)^{-1}\right)^{\frac{1}{\operatorname{ctag}-k \operatorname{tag}}} .
$$

Recover the message as $m=\frac{C_{0} \cdot A}{e\left(C_{1}, K_{2}\right) e\left(C_{2}, K_{3}\right)}$. As in the IBE, decryption can be done in a single step involving 3 pairings.

Correctness: Let $\mathcal{C} \longleftarrow I P \mathcal{E}_{1}$. Encrypt $\left(\mathcal{P P}, m, \mathbf{x}=\left(x_{1}, \ldots, x_{n}\right) ; s\right)$ where $\mathcal{C}=\left(\mathbf{x}, C_{0}, C_{1}, C_{2}, C_{3}\right.$, ctag $)$ and let $\mathcal{S K}_{\mathbf{y}} \longleftarrow \quad I_{\mathcal{P E}}$. KeyGen $(\mathcal{M S K}, \mathbf{y}=$ $\left.\left(y_{1}, \ldots, y_{n}\right) ; r\right)$ with $\mathcal{S K}_{\mathbf{y}}=\left(K_{1}, K_{2}, K_{3},\left(K_{4, i}, K_{5, i}, \operatorname{ktag}_{i}\right)_{i=2}^{n}\right)$. Suppose $\langle\mathbf{x}, \mathbf{y}\rangle=$ 0 and $\mathrm{ktag}=\sum_{i=2}^{n} x_{i} \mathrm{ktag}_{i} \neq \mathrm{ctag}$. First, we look at the computation of $A$. We have

$$
\begin{aligned}
\sum_{i=2}^{n} x_{i} K_{4, i} & =\sum_{i=2}^{n} x_{i} r\left(-u_{1,1} \frac{y_{i}}{y_{1}}+u_{1, i}+\operatorname{ktag}_{i} w_{1}\right) P_{2} \\
& =r\left(-\frac{u_{1,1}}{y_{1}} \sum_{i=2}^{n} x_{i} y_{i}+\sum_{i=2}^{n} x_{i} u_{1, i}+w_{1} \sum_{i=2}^{n} x_{i} \operatorname{ktag}_{i}\right) P_{2} \\
& =r\left(-\frac{u_{1,1}}{y_{1}}\left(\langle\mathbf{x}, \mathbf{y}\rangle-x_{1} y_{1}\right)+\left\langle\mathbf{x}, \mathbf{u}_{1}\right\rangle-x_{1} u_{1,1}+\mathrm{ktag} \cdot w_{1}\right) P_{2} \\
& =r\left(\left\langle\mathbf{x}, \mathbf{u}_{1}\right\rangle+\mathrm{ktag} \cdot w_{1}\right) P_{2} .
\end{aligned}
$$


Similarly, $\sum_{i=2}^{n} x_{i} K_{5, i}=r\left(\left\langle\mathbf{x}, \mathbf{u}_{2}\right\rangle+\mathrm{ktag} \cdot w_{1}\right) P_{2}$. Combining the two, we get

$$
e\left(C_{1}, \sum_{i=2}^{n} x_{i} K_{4, i}\right) e\left(C_{2}, \sum_{i=2}^{n} x_{i} K_{5}\right)=e\left(P_{1}, P_{2}\right)^{r s(\langle\mathbf{x}, \mathbf{u}\rangle+\operatorname{ktag} \cdot w)}
$$

implying that

$$
A=\left(e\left(C_{3}, K_{1}\right) e\left(C_{1}, \sum_{i=2}^{n} x_{i} K_{4, i}\right)^{-1} e\left(C_{2}, \sum_{i=2}^{n} x_{i} K_{5}\right)^{-1}\right)^{\frac{1}{\operatorname{ctag}-k \operatorname{tag}}}=e\left(P_{1}, P_{2}\right)^{r s w} .
$$

The second stage of decryption recovers the message as shown below.

$$
\begin{aligned}
\frac{C_{0} \cdot A}{e\left(C_{1}, K_{2}\right) e\left(C_{2}, K_{3}\right)} & =\frac{m \cdot g_{T}^{s} \cdot A}{e\left(s P_{1},\left(\alpha_{1}+r w_{1}\right) P_{2}\right) e\left(s b P_{1},\left(\alpha_{2}+r w_{2}\right) P_{2}\right)} \\
& =\frac{m \cdot e\left(P_{1}, P_{2}\right)^{\left(\alpha_{1}+b \alpha_{2}\right) s} \cdot e\left(P_{1}, P_{2}\right)^{r s w}}{e\left(P_{1}, P_{2}\right)^{\left(\alpha_{1}+b \alpha_{2}\right) s} e\left(P_{1}, P_{2}\right)^{r s w}} \\
& =m
\end{aligned}
$$

Before proving security, we describe algorithms that generate the necessary semi-functional objects for a dual system proof. These are required only in the proof.

$I_{\mathcal{P E}}$. SFEncrypt $(\mathcal{P} \mathcal{P}, \mathcal{M S K}, m, \mathbf{x}):$ Generate $\left(\mathcal{C}^{\prime}=\left(\mathbf{x}, C_{0}, C_{1}, C_{2}, C_{3}\right.\right.$, ctag $\left.)\right) \stackrel{\mathrm{R}}{\longleftarrow}$ $I \mathcal{P E} \mathcal{E}_{1}$. Encrypt $(\mathcal{P P}, m, \mathbf{x})$. Choose $\mu \stackrel{\mathrm{U}}{\longleftarrow} \mathbb{Z}_{p}$ and generate the semi-functional ciphertext components as follows.

$C_{0} \longleftarrow C_{0} \cdot e\left(P_{1}, P_{2}\right)^{\mu \alpha_{1}}$

$C_{1} \longleftarrow C_{1}+\mu P_{1}, C_{3} \longleftarrow C_{3}+\mu\left(\left\langle\mathbf{x}, \mathbf{u}_{1}\right\rangle+\operatorname{ctag} \cdot w_{1}\right)$.

Return $\mathcal{C}=\left(\mathbf{x}, C_{0}, C_{1}, C_{2}, C_{3}\right.$, ctag $)$ as the resulting semi-functional ciphertext.

$I_{\mathcal{A E}} . \mathrm{SFKeyGen}(\mathcal{P P}, \mathcal{M S K}, \mathbf{y})$ : Let $\mathcal{S K}_{\mathbf{y}}^{\prime}=\left(K_{1}, K_{2}, K_{3},\left(K_{4, i}, K_{5, i}, \operatorname{ktag}_{i}\right)_{i=2}^{n}\right)$ be obtained by running IPE 1 . KeyGen $(\mathcal{M S K}, \mathbf{y})$. Pick $\gamma \stackrel{\mathrm{U}}{\longleftarrow} \mathbb{Z}_{p}$ and modify the components of $\mathcal{S} \mathcal{K}_{\mathbf{y}}^{\prime}$ as follows:

$$
K_{2} \longleftarrow K_{2}+\gamma P_{2}, K_{3} \longleftarrow K_{3}-\frac{\gamma}{b} P_{2} .
$$

The semi-functional key given by $\mathcal{S K}_{\mathbf{y}}^{\prime}=\left(K_{1}, K_{2}, K_{3},\left(K_{4, i}, K_{5, i}, \operatorname{ktag}_{i}\right)_{i=2}^{n}\right)$ is returned as output.

For a given pair of ciphertext and key satisfying $\left(\mathrm{ktag}=\sum_{i=2}^{n} x_{i} \mathrm{ktag}_{i}\right) \neq \mathrm{ctag}$ and $\langle\mathbf{x}, \mathbf{y}\rangle=0$, decryption fails only when both are semi-functional since the message will be blinded by $e\left(P_{1}, P_{2}\right)^{\mu \gamma}$. It is easy to see that the rest of the semi-functional components get canceled.

We now prove that scheme $I \mathcal{P E} \mathcal{E}_{1}$ is adaptively secure, formalised in the theorem below.

Theorem 1. Scheme IPE 1 is $(q, \varepsilon, t)$-IND-CPA-secure if the $\left(\varepsilon_{\mathrm{DDH} 1}, t_{1}\right)$-DDH1 and $\left(\varepsilon_{\mathrm{DDH} 2}, t_{2}\right)-\mathrm{DDH} 2$ assumptions hold in the underlying pairing description $\mathcal{G}$ where $\varepsilon \leq \varepsilon_{\mathrm{DDH} 1}+q \cdot \varepsilon_{\mathrm{DDH} 2}+(1 / p)$ and $t=\max \left(t_{1}, t_{2}\right)-O(q \rho)$, $\rho$ being the maximum cost of scalar multiplication in either $\mathbb{G}_{1}$ or $\mathbb{G}_{2}$. 
Proof Sketch. Let $G_{0}$ denote the real security game ind-cpa (defined in Section 2.3). The proof proceeds though a sequence of games where we gradually change the distribution of the keys and challenge ciphertext provided to the adversary. At the end is the game where the attacker receives semi-functional encryption of a random message. We first change the ciphertext to semi-functional form and then the $q$ keys provided as answers to the $q$ queries to semi-functional form. There are essentially three main parts in the reduction.

Distinguishing normal and semi-functional ciphertexts: We show that an attacker's ability to distinguish between normal and semi-functional ciphertexts can be leveraged to solve the DDH1 problem. This is clear from the definition of semi-functional ciphertexts. $P_{1}, b P_{1}$ and $s b P_{1}$ come from the instance and are sufficient to simulate the correct environment. The DDH1 challenge is embedded in $C_{1}$ which is either normal or semi-functional according as the instance is real or random. Since no encoding of $b$ is known in $\mathbb{G}_{2}$, the simulator itself cannot create a semi-functional key and detect the type of the challenge ciphertext.

Detecting the change of $k$-th key from normal to semi-functional:

This is the most crucial stage of the security reduction. Denote by $\mathbf{y}_{1}, \ldots, \mathbf{y}_{q}$ the queries made by the attacker. The first $k-1$ keys returned are semifunctional and the last $q-k-1$ keys are normal. The simulator is designed in a way that it can create both normal and semi-functional keys. The DDH2 challenge is embedded in the $k$-th key and particularly in component $K_{2}$. However, for the $k$-th key the simulator can only create a semi-functional ciphertext with ctag $=\sum_{i=2}^{n} x_{i} \mathrm{ktag}_{i}$. This ensures that the simulator itself cannot detect the type of $k$-th key and trivially solve DDH2. Furthermore, the tags in the ciphertext and keys need to be uniformly and independently distributed in the attacker's view. This is achieved by setting them as

$$
\left(\begin{array}{c}
\widehat{\operatorname{ctag}} \\
\operatorname{ktag}_{2} \\
\vdots \\
\operatorname{ktag}_{n}
\end{array}\right)=\left(\begin{array}{ccccc}
-\widehat{x}_{1} & -\widehat{x}_{2} & -\widehat{x}_{3} & \cdots & -\widehat{x}_{n} \\
y_{2} / y_{1} & -1 & 0 & \cdots & 0 \\
y_{3} / y_{1} & 0 & -1 & \cdots & 0 \\
\vdots & \vdots & \vdots & \ddots & \vdots \\
y_{n} / y_{1} & 0 & 0 & \cdots & -1
\end{array}\right)\left(\begin{array}{c}
v_{2,1} \\
v_{2,2} \\
\vdots \\
v_{2, n}
\end{array}\right)
$$

where $\widehat{c t a g}$ is the tag associated with the challenge ciphertext for the challenge vector $\widehat{\mathbf{x}}=\left(\widehat{x}_{1}, \ldots, \widehat{x}_{n}\right)$ and $\operatorname{ktag}_{2}, \ldots, \operatorname{ktag}_{n}$ are the tags associated with the secret key for $\mathbf{y}_{k}$. The matrix has determinant $(-1)^{n}\left\langle\widehat{\mathbf{x}}, \mathbf{y}_{k}\right\rangle / y_{1}$ which is non-zero because all of $\mathscr{A}$ 's queries are such that $\left\langle\widehat{\mathbf{x}}, \mathbf{y}_{k}\right\rangle \neq 0$. (Here $y_{1}$ is the first coordinate of $\mathbf{y}_{k}$ ). Hence all we need to do is choose $\mathbf{v}_{2}=\left(v_{2,1}, \ldots, v_{2, n}\right)$ uniformly from $\mathbb{Z}_{p}^{n}$ and also hide $\mathbf{v}_{2}$ information theoretically from the attacker. $\mathbf{v}_{2}$ is in fact embedded in the master secret key (and as a result in the public parameters) but masked by other additive terms. The argument repeated $q$ times for each query gives a degradation of $q$ in DDH2.

Distinguishing the real message from a random one: The last important step is an information theoretic argument to show that the message 
encrypted is random that is, the bit $\beta$ is statistically hidden form the attacker. This is done by changing the setup and semi-functional key generation algorithms in such a way that all information provided to the attacker are independent of $\alpha_{1}$. The only component that depends on $\alpha_{1}$ is $C_{0}$ of the challenge ciphertext where the message has a blinding factor of $e\left(P_{1}, P_{2}\right)^{\mu \alpha_{1}}$. Since all other information is independent of $\alpha_{1}, m_{\beta} \cdot e\left(P_{1}, P_{2}\right)^{\mu \alpha_{1}}$ is uniformly distributed in $\mathbb{G}_{T}$ and thus provides no hint to about $\beta$ unless $\mu=0$ which happens with probability $1 / p$.

Refer to Appendix A for details of the proof.

\section{$5 \quad$ Weakly Attribute-Hiding IPE}

In this section, we present our second IPE construction $I \mathcal{A E} \mathcal{E}_{2}$ for inner products over $\mathbb{Z}_{p}^{n}$. Unlike $I \mathscr{P \mathcal { E } _ { 1 }}$, this construction is based on $\mathcal{I R}-I \mathcal{B E}$. While the $n$-equation revocation technique was used in [AL10] to obtain constantsize ciphertexts forgoing attribute-hiding, we use it here to anonymise ciphertexts by incorporating the technique into the encryption algorithm. We split the ciphertext component of $\mathscr{I R}-I \mathcal{B E}$ containing the identity hash into $n-1$ components corresponding to the entries of the attribute vector $\mathbf{x}$. For decryption, the relation $R(\mathbf{x}, \mathbf{y})$ can be verified by combining the ciphertext components using the secret vector $\mathbf{y}$ without knowing $\mathbf{x}$. Let $I P E_{2}=$ $\left(I P E_{2}\right.$.Setup, $I P E_{2}$.Encrypt, $I \mathscr{P E} \mathcal{E}_{2}$.KeyGen, $I \mathscr{P E} \mathcal{E}_{2}$. Decrypt) with the algorithms described as below.

$I \mathscr{P E} \mathcal{E}_{2}$.Setup $(\kappa, n)$ : Generate a Type-3 pairing $\mathcal{G}=\left(p, \mathbb{G}_{1}, \mathbb{G}_{2}, \mathbb{G}_{T}, e, F_{1}, F_{2}\right)$ based on the security parameter $\kappa$. Choose $P_{1} \stackrel{\mathrm{U}}{\longleftarrow} \mathbb{G}_{1}^{\times}, P_{2} \stackrel{\mathrm{U}}{\longleftarrow} \mathbb{G}_{2}^{\times}, b \stackrel{\mathrm{U}}{\longleftarrow} \mathbb{Z}_{p}^{\times}$, $\alpha_{1}, \alpha_{2}, w_{1}, w_{2} \stackrel{\mathrm{U}}{\longleftarrow} \mathbb{Z}_{p}, \mathbf{u}_{1}, \mathbf{u}_{2} \stackrel{\mathrm{U}}{\longleftarrow} \mathbb{Z}_{p}^{n}$ and set $\mathbf{u}=\mathbf{u}_{1}+b \mathbf{u}_{2}, w=w_{1}+b w_{2}$ and $g_{T}=e\left(P_{1}, P_{2}\right)^{\alpha_{1}+b \alpha_{2}}$. The parameters are given by

$$
\begin{aligned}
& \mathcal{P P}:\left(P_{1}, b P_{1}, \mathbf{u} P_{1}, w P_{1}, g_{T}\right) \\
& \mathcal{M S K}:\left(P_{2}, \alpha_{1}, \alpha_{2}, \mathbf{u}_{1}, \mathbf{u}_{2}, w_{1}, w_{2}\right)
\end{aligned}
$$

$I \mathcal{E E}_{2}$. Encrypt $\left(\mathcal{P P}, m, \mathbf{x}=\left(x_{1}, \ldots, x_{n}\right)\right):$ The ciphertext is given by the tuple $\mathcal{C}=$ $\left(C_{0}, C_{1}, C_{2},\left(C_{3, i}, \operatorname{ctag}_{i}\right)_{i=2}^{n}\right)$ where

$$
\begin{aligned}
& \left(\operatorname{ctag}_{i}\right)_{i=2}^{n}, s \stackrel{\mathrm{U}}{\longleftarrow} \mathbb{Z}_{p}, \\
& C_{0}=m \cdot\left(g_{T}\right)^{s} \text {, } \\
& C_{1}=s P_{1}, C_{2}=s b P_{1} \text {, } \\
& C_{3, i}=s\left(-\frac{x_{i}}{x_{1}} u_{1}+u_{i}+\operatorname{ctag}_{i} w\right) P_{1} \text { for } i=2, \ldots, n \text {. }
\end{aligned}
$$

Since $\left(u_{i} P_{1}\right)_{i \in[1, n]}$ and $w P_{1}$ are provided in $\mathcal{P} \mathcal{P}$, each $C_{3, i}$ can be computed using 3 scalar multiplications.

$I P E_{2} \cdot \operatorname{Key} \operatorname{Gen}\left(\mathcal{M S K}, \mathbf{y}=\left(y_{1}, \ldots, y_{n}\right)\right)$ : Secret key $\mathcal{S} \mathcal{K}_{\mathbf{y}}=\left(K_{1}, K_{2}, K_{3}, K_{4}, K_{5}\right)$ is computed as follows. 


$$
\begin{aligned}
& r \stackrel{\mathrm{U}}{\overleftarrow{ }} \mathbb{Z}_{p}, \\
& K_{1}=r P_{2}, K_{2}=\left(\alpha_{1}+r\left\langle\mathbf{y}, \mathbf{u}_{1}\right\rangle\right) P_{2}, K_{3}=\left(\alpha_{2}+r\left\langle\mathbf{y}, \mathbf{u}_{2}\right\rangle\right) P_{2} \\
& K_{4}=r w_{1} P_{2}, K_{5}=r w_{2} P_{2} .
\end{aligned}
$$

$I \mathcal{P E}_{2}$. Decrypt $\left(\mathcal{C}, \mathcal{S K}_{\mathbf{y}}, \mathbf{y}\right)$ : Compute ctag $=\sum_{i=2}^{n} y_{i} \operatorname{ctag}_{i}$. Recover the message as follows.

$$
m=\frac{C_{0} \cdot e\left(\sum_{i=2}^{n} y_{i} C_{3, i}, K_{1}\right)}{e\left(C_{1}, K_{2}+\operatorname{ctag} K_{4}\right) e\left(C_{2}, K_{3}+\operatorname{ctag} K_{5}\right)} .
$$

Correctness. Let $\mathcal{C} \stackrel{\mathrm{R}}{\longleftarrow} I \mathcal{P E}_{2}$. Encrypt $\left(\mathcal{P P}, m, \mathbf{x}=\left(x_{1}, \ldots, x_{n}\right) ; s\right)$ and let $\mathcal{S} \mathcal{K}_{\mathbf{y}} \stackrel{\mathrm{R}}{\longleftarrow} I \mathcal{P E}_{2} . \operatorname{Key} \operatorname{Gen}\left(\mathcal{M S K}, \mathbf{y}=\left(y_{1}, \ldots, y_{n}\right) ; r\right)$ where $\mathcal{C}, \mathcal{S} \mathcal{K}_{\mathbf{y}}$ are given by $\left(C_{0}, C_{1}, C_{2},\left(C_{3, i}, \operatorname{ctag}_{i}\right)_{i=2}^{n}\right), \mathcal{S} \mathcal{K}_{\mathbf{y}}=\left(K_{1}, K_{2}, K_{3}, K_{4}, K_{5}\right)$ respectively. Suppose $\langle\mathbf{x}, \mathbf{y}\rangle=0$ and $\operatorname{ctag}=\sum_{i=2}^{n} y_{i} \operatorname{ctag}_{i}$. Let $A_{1}=e\left(\sum_{i=2}^{n} y_{i} C_{3, i}, K_{1}\right)$ and $A_{2}=$ $e\left(C_{1}, K_{2}+\operatorname{ctag} K_{4}\right) e\left(C_{2}, K_{3}+\operatorname{ctag} K_{5}\right)$. Decryption is correct if $A_{2} / A_{1}=\left(g_{T}\right)^{s}$. We have

$$
\begin{aligned}
A_{1} & =e\left(\sum_{i=2}^{n} y_{i} C_{3, i}, K_{1}\right) \\
& =e\left(\sum_{i=2}^{n} y_{i} s\left(-\frac{x_{i} u_{1}}{x_{1}}+u_{i}+\operatorname{ctag}_{i} w\right) P_{1}, r P_{2}\right) \\
& =e\left(\left(-\left(\langle\mathbf{y}, \mathbf{x}\rangle-x_{1} y_{1}\right) \frac{u_{1}}{x_{1}}+\langle\mathbf{y}, \mathbf{u}\rangle-y_{1} u_{1}+\operatorname{ctag} \cdot w\right) P_{1}, P_{2}\right)^{r s} \\
& =e\left(P_{1}, P_{2}\right)^{r s(\langle\mathbf{y}, \mathbf{u}\rangle+\operatorname{ctag} \cdot w)},
\end{aligned}
$$

and

$$
\begin{aligned}
A_{2} & =e\left(C_{1}, K_{2}+\operatorname{ctag} K_{4}\right) e\left(C_{2}, K_{3}+\operatorname{ctag} K_{5}\right) \\
& =e\left(s P_{1},\left(\alpha_{1}+r\left\langle\mathbf{y}, \mathbf{u}_{1}\right\rangle\right) P_{2}+\operatorname{ctag} \cdot r w_{1} P_{2}\right) e\left(s b P_{1}\left(\alpha_{2}+r\left\langle\mathbf{y}, \mathbf{u}_{2}\right\rangle\right) P_{2}+\operatorname{ctag} \cdot r w_{2} P_{2}\right) \\
& =e\left(P_{1},\left(\alpha_{1}+b \alpha_{2}\right) P_{2}\right)^{s} e\left(P_{1}, r\left(\left\langle\mathbf{y}, \mathbf{u}_{1}\right\rangle+b\left\langle\mathbf{y}, \mathbf{u}_{2}\right\rangle+\operatorname{ctag}\left(w_{1}+b w_{2}\right)\right) P_{2}\right)^{s} \\
& =\left(g_{T}\right)^{s} \cdot e\left(P_{1},\left(\left\langle\mathbf{y}, \mathbf{u}_{1}+b \mathbf{u}_{2}\right\rangle+\operatorname{ctag} \cdot w\right) P_{2}\right)^{r s} \\
& =\left(g_{T}\right)^{s} \cdot e\left(P_{1}, P_{2}\right)^{r s(\langle\mathbf{y}, \mathbf{u}\rangle+\operatorname{ctag} \cdot w)}
\end{aligned}
$$

thus implying that $A_{2} / A_{1}=\left(g_{T}\right)^{s}$, as desired.

Security. The theorem below summarises the security guarantee we obtain for $I P \mathcal{E}_{2}$.

Theorem 2. Scheme IPE $\mathcal{I}_{2}$ is $(q, \varepsilon, t)$-IND-WAH-CPA-secure if the $\left(\varepsilon_{\mathrm{DDH} 1}, t_{1}\right)$ DDH1 and $\left(\varepsilon_{\mathrm{DDH} 2}, t_{2}\right)$-DDH2 assumptions hold in the underlying pairing description $\mathcal{G}$ where $\varepsilon \leq \varepsilon_{\mathrm{DDH} 1}+q \cdot \varepsilon_{\mathrm{DDH} 2}+(1 / p)$ and $t=\max \left(t_{1}, t_{2}\right)-O(q \rho)$, $\rho$ being the maximum cost of scalar multiplication in either $\mathbb{G}_{1}$ or $\mathbb{G}_{2}$.

The proof is more or less similar to the proof of Theorem 1 except for the information theoretic argument in the last step. In addition to showing that the blinding factor on the message is uniformly random in the attacker's view, 
we also need to prove that the attribute vector is hidden from the adversary. The solution is to simulate the key extraction queries in such a way that all information the attacker sees is independent of $\mathbf{u}_{1}$. Observe that $\mathbf{u}_{1}$ is part of the master secret and would also be used to define the semi-functional components for $C_{3, i}$. With all keys and parameters being independent of $\mathbf{u}_{1}$, one can argue that $C_{3, i}$ components are uniform and independent elements of $\mathbb{G}_{1}$ thus providing no hint about which attribute vector the challenge ciphertext is encrypted to. (This makes sense as the only ciphertext components determined by the attribute vector are $C_{3, i}$ for $\left.i=2, \ldots, n\right)$. A detailed proof is provided in Appendix B.

\section{Acknowledgements}

I would like to thank Benoit Libert and Palash Sarkar for helpful discussions as well as the reviewers of ACNS'16 for their valuable comments. This research was funded by the "Programme Avenir Lyon Saint-Etienne de l'Universite de Lyon" in the framework of the programme "Investissements d'Avenir" (ANR11-IDEX-0007).

\section{References}

[AC16] Shashank Agrawal and Melissa Chase. A study of pair encodings: Predicate encryption in prime order groups. In Eyal Kushilevitz and Tal Malkin, editors, Theory of Cryptography - 13th International Conference, TCC 2016A, Tel Aviv, Israel, January 10-13, 2016, Proceedings, Part II, volume 9563 of Lecture Notes in Computer Science, pages 259-288. Springer, 2016.

[AL10] Nuttapong Attrapadung and Benoît Libert. Functional encryption for inner product: Achieving constant-size ciphertexts with adaptive security or support for negation. In Phong Q. Nguyen and David Pointcheval, editors, Public Key Cryptography - PKC 2010, 13th International Conference on Practice and Theory in Public Key Cryptography, Paris, France, May 2628, 2010. Proceedings, volume 6056 of Lecture Notes in Computer Science, pages 384-402. Springer, 2010.

[Att14] Nuttapong Attrapadung. Dual system encryption via doubly selective security: Framework, fully secure functional encryption for regular languages, and more. In Phong Q. Nguyen and Elisabeth Oswald, editors, Advances in Cryptology - EUROCRYPT 2014 - 33rd Annual International Conference on the Theory and Applications of Cryptographic Techniques, Copenhagen, Denmark, May 11-15, 2014. Proceedings, volume 8441 of Lecture Notes in Computer Science, pages 557-577. Springer, 2014.

[Att15] Nuttapong Attrapadung. Dual system encryption framework in prime-order groups. IACR Cryptology ePrint Archive, 2015:390, 2015.

[BKP14] Olivier Blazy, Eike Kiltz, and Jiaxin Pan. (hierarchical) identity-based encryption from affine message authentication. In Juan A. Garay and Rosario Gennaro, editors, CRYPTO (1), volume 8616 of Lecture Notes in Computer Science, pages 408-425. Springer, 2014.

[Boy13] Xavier Boyen. Attribute-Based Functional Encryption on Lattices. In TCC, pages $122-142,2013$. 
[BSW07] John Bethencourt, Amit Sahai, and Brent Waters. Ciphertext-Policy Attribute-Based Encryption. In IEEE Symposium on Security and Privacy, pages 321-334. IEEE Computer Society, 2007.

[CG13] Ran Canetti and Juan A. Garay, editors. Advances in Cryptology - CRYPTO 2013 - 33rd Annual Cryptology Conference, Santa Barbara, CA, USA, August 18-22, 2013. Proceedings, Part II, volume 8043 of Lecture Notes in Computer Science. Springer, 2013.

[CGW15] Jie Chen, Romain Gay, and Hoeteck Wee. Improved dual system ABE in prime-order groups via predicate encodings. In Elisabeth Oswald and Marc Fischlin, editors, Advances in Cryptology - EUROCRYPT 2015 - 34th Annual International Conference on the Theory and Applications of Cryptographic Techniques, Sofia, Bulgaria, April 26-30, 2015, Proceedings, Part II, volume 9057 of Lecture Notes in Computer Science, pages 595-624. Springer, 2015.

[CW13] Jie Chen and Hoeteck Wee. Fully, (almost) tightly secure IBE and dual system groups. In Canetti and Garay [CG13], pages 435-460. Full version available as IACR Technical Report, 2013/803, http://eprint.iacr.org/ 2013/803.

$\left[\mathrm{GGH}^{+} 13\right]$ Sanjam Garg, Craig Gentry, Shai Halevi, Amit Sahai, and Brent Waters. Attribute-based encryption for circuits from multilinear maps. In Canetti and Garay [CG13], pages 479-499.

[GPSW06] Vipul Goyal, Omkant Pandey, Amit Sahai, and Brent Waters. Attributebased encryption for fine-grained access control of encrypted data. In Ari Juels, Rebecca N. Wright, and Sabrina De Capitani di Vimercati, editors, ACM Conference on Computer and Communications Security, pages 89-98. ACM, 2006.

[GVW13] Sergey Gorbunov, Vinod Vaikuntanathan, and Hoeteck Wee. Attributebased encryption for circuits. In Dan Boneh, Tim Roughgarden, and Joan Feigenbaum, editors, Symposium on Theory of Computing Conference, STOC'13, Palo Alto, CA, USA, June 1-4, 2013, pages 545-554. ACM, 2013.

[GW09] Craig Gentry and Brent Waters. Adaptive security in broadcast encryption systems (with short ciphertexts). In Antoine Joux, editor, EUROCRYPT, volume 5479 of Lecture Notes in Computer Science, pages 171-188. Springer, 2009.

[JR13] Charanjit S. Jutla and Arnab Roy. Shorter quasi-adaptive NIZK proofs for linear subspaces. In Kazue Sako and Palash Sarkar, editors, ASIACRYPT (1), volume 8269 of Lecture Notes in Computer Science, pages 1-20. Springer, 2013.

[JR15] Charanjit S. Jutla and Arnab Roy. Dual-system simulation-soundness with applications to UC-PAKE and more. In Tetsu Iwata and Jung Hee Cheon, editors, Advances in Cryptology - ASIACRYPT 2015 - 21st International Conference on the Theory and Application of Cryptology and Information Security, Auckland, New Zealand, November 29 - December 3, 2015, Proceedings, Part I, volume 9452 of Lecture Notes in Computer Science, pages 630-655. Springer, 2015.

[KSW08] Jonathan Katz, Amit Sahai, and Brent Waters. Predicate Encryption Supporting Disjunctions, Polynomial Equations, and Inner Products. In Nigel P. Smart, editor, EUROCRYPT, volume 4965 of Lecture Notes in Computer Science, pages 146-162. Springer, 2008.

[LSW08] Allison B. Lewko, Amit Sahai, and Brent Waters. Revocation systems with very small private keys. IACR Cryptology ePrint Archive, 2008:309, 2008. 
[LW12] Allison Lewko and Brent Waters. New Proof Methods for Attribute-Based Encryption: Achieving Full Security through Selective Techniques. In Reihaneh Safavi-Naini and Ran Canetti, editors, CRYPTO, volume 7417 of Lecture Notes in Computer Science, pages 180-198. Springer, 2012.

[OSW07] Rafail Ostrovsky, Amit Sahai, and Brent Waters. Attribute-based encryption with non-monotonic access structures. In Peng Ning, Sabrina De Capitani di Vimercati, and Paul F. Syverson, editors, ACM Conference on Computer and Communications Security, pages 195-203. ACM, 2007.

[OT08] Tatsuaki Okamoto and Katsuyuki Takashima. Homomorphic Encryption and Signatures from Vector Decomposition. In Steven D. Galbraith and Kenneth G. Paterson, editors, Pairing, volume 5209 of Lecture Notes in Computer Science, pages 57-74. Springer, 2008.

[OT09] Tatsuaki Okamoto and Katsuyuki Takashima. Hierarchical Predicate Encryption for Inner-Products. In Mitsuru Matsui, editor, ASIACRYPT, volume 5912 of Lecture Notes in Computer Science, pages 214-231. Springer, 2009.

[OT10] Tatsuaki Okamoto and Katsuyuki Takashima. Fully Secure Functional Encryption with General Relations from the Decisional Linear Assumption. In Tal Rabin, editor, CRYPTO, volume 6223 of Lecture Notes in Computer Science, pages 191-208. Springer, 2010.

[OT11] Tatsuaki Okamoto and Katsuyuki Takashima. Achieving Short Ciphertexts or Short Secret-Keys for Adaptively Secure General Inner-Product Encryption. In Dongdai Lin, Gene Tsudik, and Xiaoyun Wang, editors, CANS, volume 7092 of Lecture Notes in Computer Science, pages 138-159. Springer, 2011.

[RS14a] Somindu C. Ramanna and Palash Sarkar. Efficient adaptively secure IBBE from standard assumptions. IACR Cryptology ePrint Archive, 2014:380, 2014. To appear in IEEE Transactions on Information Theory.

[RS14b] Somindu C. Ramanna and Palash Sarkar. Efficient (anonymous) compact HIBE from standard assumptions. In Sherman S. M. Chow, Joseph K. Liu, Lucas Chi Kwong Hui, and Siu-Ming Yiu, editors, Provable Security - 8th International Conference, ProvSec 2014, Hong Kong, China, October 9-10, 2014. Proceedings, volume 8782 of Lecture Notes in Computer Science, pages 243-258. Springer, 2014.

[SW05] Amit Sahai and Brent Waters. Fuzzy Identity-Based Encryption. In Ronald Cramer, editor, EUROCRYPT, volume 3494 of Lecture Notes in Computer Science, pages 457-473. Springer, 2005.

[Wat09] Brent Waters. Dual System Encryption: Realizing Fully Secure IBE and HIBE under Simple Assumptions. In Shai Halevi, editor, CRYPTO, volume 5677 of Lecture Notes in Computer Science, pages 619-636. Springer, 2009.

[Wat11] Brent Waters. Ciphertext-Policy Attribute-Based Encryption: An Expressive, Efficient, and Provably Secure Realization. In Dario Catalano, Nelly Fazio, Rosario Gennaro, and Antonio Nicolosi, editors, Public Key Cryptography, volume 6571 of Lecture Notes in Computer Science, pages 53-70. Springer, 2011.

[Wee14] Hoeteck Wee. Dual system encryption via predicate encodings. In Yehuda Lindell, editor, Theory of Cryptography - 11th Theory of Cryptography Conference, TCC 2014, San Diego, CA, USA, February 24-26, 2014. Proceedings, volume 8349 of Lecture Notes in Computer Science, pages 616-637. Springer, 2014. 


\section{A Proof of Theorem 1}

The proof is a hybrid argument over a sequence of $q+6$ games $\mathrm{G}_{0}, \mathrm{G}_{1}$, $\mathrm{G}_{2}, \mathrm{G}_{3,0}, \ldots, \mathrm{G}_{3, q}, \mathrm{G}_{4}, \mathrm{G}_{5}$ where $\mathrm{G}_{0}$ is the real ind-cpa attack game defined in Section 2.3. Let $X_{\square}$ denote the event that the adversary $\mathscr{A}$ wins (i.e., $\beta=\beta^{\prime}$ ) in $\mathrm{G}_{\square}$. The rest of the games are described below.

Game $G_{1}$ : This game is similar to $G_{0}$ except for a modification to the Encrypt algorithm. Elements from the master secret key $\mathcal{M S K}$ are used instead of the public parameters. The challenge ciphertext is generated as follows.

$$
\begin{aligned}
& \text { ctag, } s \stackrel{\mathrm{U}}{\longleftarrow} \mathbb{Z}_{p} \text {, } \\
& C_{1}=s P_{1}, C_{2}=s b P_{1}, C_{3}=\left(\left\langle\widehat{\mathbf{x}}, \mathbf{u}_{1}\right\rangle+\mathrm{ctag} \cdot w_{1}\right) C_{1}+\left(\left\langle\widehat{\mathbf{x}}, \mathbf{u}_{2}\right\rangle+\mathrm{ctag} \cdot w_{2}\right) C_{2} . \\
& C_{0}=m_{\beta} \cdot\left(C_{1}, P_{2}\right)^{\alpha_{1}} e\left(C_{2}, P_{2}\right)^{\alpha_{2}} \text {, }
\end{aligned}
$$

It is straightforward to verify that $C_{0}$ and $C_{3}$ are well-formed. The change we have made is only conceptual and hence $G_{1}$ is identical to $G_{0}$. Therefore, we have

$$
\operatorname{Pr}\left[X_{0}\right]=\operatorname{Pr}\left[X_{1}\right] .
$$

Game $G_{2}$ : The challenge ciphertext generation in $G_{1}$ is further modified to obtain game $\mathrm{G}_{2}$. Component $C_{1}$ is generated as $C_{1} \stackrel{\mathrm{U}}{\longleftarrow} \mathbb{G}_{1}$. The rest of the steps remain the same. We show that the ability of the adversary to detect this change can be used to construct a DDH1-solver. More precisely we have the following.

Lemma 1. $\left|\operatorname{Pr}\left[X_{1}\right]-\operatorname{Pr}\left[X_{2}\right]\right| \leq \varepsilon_{\mathrm{DDH} 1}$.

Game $G_{3,0}$ : We arrive at $G_{3,0}$ by modifying the setup, key generation and encryption procedures in $G_{2}$ as follows.

Setup: The public parameters are generated as follows. Choose $P_{1} \longleftarrow$ $\mathbb{G}_{1}^{\times}, P_{2} \stackrel{\mathrm{U}}{\longleftarrow} \mathbb{G}_{2}^{\times}, \alpha, \alpha_{1}, w, w_{1} \stackrel{\mathrm{U}}{\longleftarrow} \mathbb{Z}_{p}, b \stackrel{\mathrm{U}}{\longleftarrow} \mathbb{Z}_{p}^{\times}, \mathbf{u}, \mathbf{u}_{1} \stackrel{\mathrm{U}}{\longleftarrow} \mathbb{Z}_{p}^{n}$, implicitly setting $\mathbf{u}_{2}=b^{-1}\left(\mathbf{u}-\mathbf{u}_{1}\right), w_{2}=b^{-1}\left(w-w_{1}\right)$ and $\alpha_{2}=b^{-1}\left(\alpha-\alpha_{1}\right)$. Set $g_{T}=e\left(P_{1}, P_{2}\right)^{\alpha}$ and $\mathcal{P} \mathcal{P}=\left(P_{1}, b P_{1}, \mathbf{u} P_{1}, w P_{1}, g_{T}\right)$.

Key Generation: On input a vector $\mathbf{y}$, the KeyGen algorithm is modified to create the components of $\mathcal{S} \mathcal{K}_{\mathbf{y}}$ as shown below.

$$
\begin{aligned}
& r,\left(\operatorname{ktag}_{i}\right)_{i=2}^{n} \stackrel{\mathrm{U}}{\longleftarrow} \mathbb{Z}_{p}, \\
& K_{1}=r P_{2}, K_{2}=\left(\alpha_{1}+r w_{1}\right) P_{2}, K_{3}=b^{-1}\left((\alpha+r w) P_{2}-K_{2}\right) \\
& \text { For } i=2, \ldots, n \text {, } \\
& K_{4, i}=r\left(-u_{1,1} \frac{y_{i}}{y_{1}}+u_{1, i}+\operatorname{ktag}_{i} w_{1}\right) P_{2}, \\
& K_{5, i}=b^{-1}\left(r\left(-u_{1} \frac{y_{i}}{y_{1}}+u_{i}+\operatorname{ktag}_{i} w\right) P_{2}-K_{4, i}\right) \text {. }
\end{aligned}
$$

Encryption: The Encrypt algorithm is modified to use public parameters instead of elements from the master secret (as in the construction). Further, the challenge ciphertext is generated by a call to the SFEncrypt algorithm. Essentially, the components of $\widehat{\mathcal{C}}$ are generated as follows. 


$$
\begin{aligned}
& \text { ctag, } s, \mu \stackrel{\mathrm{U}}{\overleftarrow{Z}} \mathbb{Z}_{p}, \\
& C_{0}=m_{\beta} \cdot\left(g_{T}\right)^{s} e\left(P_{1}, P_{2}\right)^{\mu \alpha_{1}}, \\
& C_{1}=s P_{1}+\mu P_{1}, C_{2}=s b P_{1}, \\
& C_{3}=s(\langle\mathbf{x}, \mathbf{u}\rangle+\operatorname{ctag} \cdot w) P_{1}+\mu\left(\left\langle\mathbf{x}, \mathbf{u}_{1}\right\rangle+\operatorname{ctag} \cdot w_{1}\right) P_{1} .
\end{aligned}
$$

It can be shown that the keys and $\widehat{\mathcal{C}}$ have the right form via simple calculations. The changes we have made are only conceptual. Therefore, game $G_{3,0}$ is identical to $\mathrm{G}_{2}$ and we have

$$
\operatorname{Pr}\left[X_{2}\right]=\operatorname{Pr}\left[X_{3,0}\right]
$$

Game $\mathrm{G}_{3, k}$ (for $\left.k=1, \ldots, q\right)$ : Let $\mathbf{y}_{1}, \ldots, \mathbf{y}_{q}$ be the vectors queried by $\mathscr{A}$. This game is identical to $\mathrm{G}_{3, k-1}$ except for the following two modifications. The $(k-1)$-st key is generated according to the SFKeyGen algorithm and $\mathcal{S K}_{\mathbf{y}_{k}}$ for the $k$-th vector $\mathbf{y}_{k}$ is generated differently - component $K_{2}$ of $\mathcal{S K}_{\mathbf{y}_{k}}$ is now chosen uniformly at random from $\mathbb{G}_{2}$. The first change is purely conceptual and hence does not affect the adversary's behaviour in any way. We show that if the adversary can detect the second modification, then the DDH2 problem can be solved. We have the following lemma.

Lemma 2. $\left|\operatorname{Pr}\left[X_{3, k-1}\right]-\operatorname{Pr}\left[X_{3, k}\right]\right| \leq \varepsilon_{\mathrm{DDH} 2}$ for all $k \in[1, q]$.

Game $\mathrm{G}_{4}$ : Proceeds identical to $\mathrm{G}_{3, q}$ but for one difference - the secret key corresponding to $\mathbf{y}_{q}$ is computed using the SFKeyGen algorithm. Since this is only a conceptual change, we have

$$
\operatorname{Pr}\left[X_{3, q}\right]=\operatorname{Pr}\left[X_{4}\right]
$$

Game $\mathrm{G}_{5}$ : This game is similar to $\mathrm{G}_{4}$ except for a modification of the SFKeyGen algorithm. The setup remains same as defined in $\mathrm{G}_{3,0}$. Let $\mathbf{y}$ be the input to SFKeyGen.

$$
\begin{aligned}
& r, \gamma^{\prime} \stackrel{\mathrm{U}}{\longleftarrow} \mathbb{Z}_{p} \\
& K_{1}=r P_{2}, K_{2}=\left(\gamma^{\prime}+r w_{1}\right) P_{2}, K_{3}=b^{-1}\left((\alpha+r w) P_{2}-K_{2}\right)
\end{aligned}
$$

The rest of the secret key components are generated as in $\mathrm{G}_{3,0}$. This implicitly sets $\gamma^{\prime}=\alpha_{1}+\gamma$ and induces a uniform distribution on $\gamma$. Furthermore, all keys are computed independent of $\alpha_{1}$ and so are the public parameters. As a result, in the adversary's view, $e\left(P_{1}, P_{2}\right)^{\mu \alpha_{1}}$ is randomly distributed in $\mathbb{G}_{T}$ as long as $\mu \neq 0$ which happens with probability at most $1 / p$. Hence

$$
\left|\operatorname{Pr}\left[X_{4}\right]-\operatorname{Pr}\left[X_{5}\right]\right| \leq(1 / p)
$$

The probability that $\mathscr{A}$ wins in $\mathrm{G}_{5}$, where $m_{\beta}$ is masked by $e\left(P_{1}, P_{2}\right)^{\mu \alpha_{1}}$, is exactly $1 / 2$ as the bit $b$ is information theoretically hidden from the attacker. 
Therefore, from Lemmas 1, 2 and equations (1), (2), (3) and (4), we have

$$
\begin{aligned}
\varepsilon= & \left|\operatorname{Pr}\left[X_{0}\right]-\frac{1}{2}\right| \\
= & \left|\operatorname{Pr}\left[X_{0}\right]-\operatorname{Pr}\left[X_{5}\right]\right| \\
\leq & \left|\operatorname{Pr}\left[X_{0}\right]-\operatorname{Pr}\left[X_{1}\right]\right|+\left|\operatorname{Pr}\left[X_{1}\right]-\operatorname{Pr}\left[X_{2}\right]\right|+\left|\operatorname{Pr}\left[X_{2}\right]-\operatorname{Pr}\left[X_{3}, 0\right]\right| \\
& +\left(\sum_{k=1}^{q}\left|\operatorname{Pr}\left[X_{3, k-1}\right]-\operatorname{Pr}\left[X_{3, k}\right]\right|\right)+\left|\operatorname{Pr}\left[X_{3, q}\right]-\operatorname{Pr}\left[X_{4}\right]\right|+\left|\operatorname{Pr}\left[X_{4}\right]-\operatorname{Pr}\left[X_{5}\right]\right| \\
\leq & \varepsilon_{\mathrm{DDH} 1}+q \cdot \varepsilon_{\mathrm{DDH} 2}+(1 / p)
\end{aligned}
$$

Proof (of Lemma 1). We build an algorithm $\mathscr{B}$ that can solve DDH1 if the adversary $\mathscr{A}$ is able to distinguish between games $\mathrm{G}_{1}$ and $\mathrm{G}_{2}$. Let $\left(\mathcal{G}, P_{1}, b P_{1}, s b P_{1},(s+\right.$ $\mu) P_{1}$ ) be a DDH1 instance given to $\mathscr{B}$. The task here is to decide whether $\mu=0$ or $\mu \stackrel{\mathrm{U}}{\longleftarrow} \mathbb{Z}_{p}$. $\mathscr{B}$ simulates different phases of the game for $\mathscr{A}$ as follows.

Setup: The group description is same as $\mathcal{G}$ of the instance. Pick $p_{2} \stackrel{\mathrm{U}}{\longleftarrow} \mathbb{G}_{2}^{\times}$, $\mathbf{u}_{1}, \mathbf{u}_{2} \stackrel{\mathrm{U}}{\longleftarrow} \mathbb{Z}_{p}^{n}, \alpha_{1}, \alpha_{2}, w_{1}, w_{2} \stackrel{\mathrm{U}}{\longleftarrow} \mathbb{Z}_{p}^{n}$ and generate the public parameters as: $\mathbf{u} P_{1}=\mathbf{u}_{1} P_{1}+\mathbf{u}_{2}\left(b P_{1}\right), w P_{1}=w_{1} P_{1}+w_{2}\left(b P_{1}\right), g_{T}=e\left(P_{1}, P_{2}\right)^{\alpha_{1}} e\left(b P_{1}, P_{2}\right)^{\alpha_{2}}$, where $b P_{1}$ comes from the DDH1 instance.

Key Generation: Keys are generated normally via the KeyGen algorithm. $\mathscr{B}$ does not know an encoding of $b$ in $\mathbb{G}_{2}$ and hence cannot create a semi-functional key and trivially win the game.

Challenge: $\mathscr{A}$ sends two messages $m_{0}, m_{1}$ and attribute vector $\widehat{\mathbf{x}}$ to $\mathscr{B}$. Pick $\beta \stackrel{\mathrm{U}}{\longleftarrow}\{0,1\}$ and generate $\widehat{\mathcal{C}}=\left(C_{0}, C_{1}, C_{2}, C_{3}, \widehat{\mathrm{ctag}}\right)$ as:

$$
\begin{aligned}
& \widehat{\mathrm{ctag}} \stackrel{\mathrm{U}}{\longleftarrow} \mathbb{Z}_{p}, \\
& C_{0}=m_{\beta} \cdot e\left(C_{1}, P_{2}\right)^{\alpha_{1}} \cdot e\left(C_{2}, P_{2}\right)^{\alpha_{2}}, \\
& C_{1}=(s+\mu) P_{1}, C_{2}=s b P_{1}, C_{3}=\left(\left\langle\mathbf{x}, \mathbf{u}_{1}\right\rangle+\operatorname{ctag} w_{1}\right) C_{1}+\left(\left\langle\mathbf{x}, \mathbf{u}_{2}\right\rangle+\operatorname{ctag} w_{2}\right) C_{2},
\end{aligned}
$$

where $s b P_{1}$ comes from the DDH1 instance. $\widehat{\mathcal{C}}$ is distributed normally if $\mu=0$ and as a ciphertext in $\mathrm{G}_{2}$ in case $\mu \stackrel{\mathrm{U}}{\longleftarrow} \mathbb{Z}_{p}$. This is because $C_{1}$ is uniformly distributed in group $\mathbb{G}_{1}$ if $\mu \stackrel{\mathrm{U}}{\longleftarrow} \mathbb{Z}_{p}$. $\mathscr{A}$ returns a bit $\beta^{\prime}$ as its guess of $\beta$ to $\mathscr{B}$. If $\mathscr{A}$ wins (i.e., $\beta=\beta^{\prime}$ ) $\mathscr{B}$ returns 1 and otherwise it returns 0 . We have,

$$
\begin{aligned}
\left|\operatorname{Pr}\left[X_{1}\right]-\operatorname{Pr}\left[X_{2}\right]\right| & =\mid \operatorname{Pr}\left[\beta=\beta^{\prime} \text { in } G_{1}\right]-\operatorname{Pr}\left[\beta=\beta^{\prime} \text { in } G_{2}\right] \mid \\
& =\left|\operatorname{Pr}\left[\beta=\beta^{\prime} \mid \mu=0\right]-\operatorname{Pr}\left[\beta=\beta^{\prime} \mid \mu \stackrel{\mathrm{U}}{\longleftarrow} \mathbb{Z}_{p}\right]\right| \\
& =\mid \operatorname{Pr}[\mathscr{B} \text { returns } 1 \mid \mu=0]-\operatorname{Pr}\left[\mathscr{B} \text { returns } 1 \mid \mu \stackrel{\mathrm{U}}{\longleftarrow} \mathbb{Z}_{p}\right] \mid \\
& =\operatorname{Adv}_{\mathcal{G}}^{D D H 1}(\mathscr{A}) \\
& \leq \varepsilon_{\mathrm{DDH} 1}
\end{aligned}
$$


Proof (of Lemma 2). If the attacker can distinguish between the two games $\mathrm{G}_{3, k-1}$ and $\mathrm{G}_{3, k}$, then we show how to build an algorithm $\mathscr{B}$ that solves the DDH2 problem. Let $\left(\mathcal{G}, P_{2}, r P_{2}, w_{1} P_{2},\left(r w_{1}+\gamma\right) P_{2}\right)$ be an instance of DDH2 provided to $\mathscr{B}$ whose task is to determine whether $\gamma=0$ or $\gamma \stackrel{\mathrm{U}}{\longleftarrow} \mathbb{Z}_{p}$. The different phases of the game are simulated as follows.

Setup: Choose $P_{1} \stackrel{U}{\longleftarrow} \mathbb{G}_{1}^{\times}, \mathbf{v}_{1}, \mathbf{v}_{2} \stackrel{\mathrm{U}}{\longleftarrow} \mathbb{Z}_{p}^{n}$ and implicitly set $\mathbf{u}_{1}=\mathbf{v}_{1}+w_{1} \mathbf{v}_{2}$. Observe that no encoding of $\mathbf{u}_{1}$ is available in $\mathbb{G}_{1}$. Nevertheless, the public parameters can be generated without using $\mathbf{u}_{1}$ or $w_{1}$, as described in $\mathrm{G}_{3,0}$.

Key Generation: Denote by $\mathbf{y}_{1}, \ldots, \mathbf{y}_{q}$ the vectors queried by $\mathscr{A}$. Normal keys can be generated as in $\mathrm{G}_{3,0}$. Note that $\mathscr{B}$ knows $w_{1} P_{2}$ and hence can compute $\mathbf{u}_{1} P_{2}$ which is required for generating components of a normal key. For $j=1, \ldots, q$, key $\mathcal{S} \mathcal{K}_{\mathbf{y}_{j}}$ is generated as follows.

Case $j<k$ : Use SFKeyGen to generate $\mathcal{S K}_{\mathbf{y}_{j}}$. This is possible since $b$ is known to $\mathscr{B}$.

Case $j=k$ : Create the key $\mathcal{S K}_{\mathbf{y}_{k}}=\left(K_{1}, K_{2}, K_{3},\left(K_{4, i}, K_{5, i}, \operatorname{ktag}_{i}\right)_{i=2}^{n}\right)$ as follows. Let $\mathbf{y}_{k}=\left(y_{1}, \ldots, y_{n}\right)$.

For $i=2, \ldots, n$, set $\operatorname{ktag}_{i}=\left(y_{i} / y_{1}\right) v_{2,1}-v_{2, i} ;$ pick $r \stackrel{\mathrm{U}}{\longleftarrow} \mathbb{Z}_{p}$,

$K_{1}=r P_{2}$,

$K_{2}=\alpha_{1} P_{2}+\left(r w_{1}+\gamma\right) P_{2}, K_{3}=b^{-1}\left(\left(\alpha-\alpha_{1}\right) P_{2}+w r P_{2}-\left(r w_{1}+\gamma\right) P_{2}\right)$,

for $i=2, \ldots, n$,

$K_{4, i}=\left(-\frac{y_{i}}{y_{1}} v_{1,1}+v_{1, i}\right) r P_{2}, K_{5, i}=b^{-1}\left(\left(-\frac{y_{i}}{y_{1}} u_{1}+u_{i}\right) P_{2}-K_{4, i}\right)$.

Case $j>k: \mathscr{B}$ computes a normal key according to the KeyGen algorithm.

The following calculation shows that $K_{4, i}$ is well-formed.

$$
\begin{aligned}
K_{4, i} & =\left(-\frac{y_{i}}{y_{1}} u_{1,1}+u_{1, i}+\operatorname{ktag}_{i} w_{1}\right) r P_{2} \\
& =\left(-\frac{y_{i}}{y_{1}}\left(v_{1,1}+w_{1} v_{2,1}\right)+\left(v_{1, i}+w_{1} v_{2, i}\right)+\left(\frac{y_{i}}{y_{1}} v_{2,1}-v_{2, i}\right) w_{1}\right) r P_{2} \\
& =\left(-\frac{y_{i}}{y_{1}} v_{1,1}+v_{1, i}\right) r P_{2}
\end{aligned}
$$

Also, for a vector $\mathbf{x}$ that is orthogonal to $\mathbf{y}_{k}$, we have

$$
\begin{aligned}
\text { ktag } & =\sum_{i=2}^{n} x_{i} \operatorname{ktag}_{i} \\
& =\sum_{i=2}^{n} x_{i}\left(\left(y_{i} / y_{1}\right) v_{2,1}-v_{2, i}\right) \\
& =\left(v_{2,1} / y_{1}\right) \sum_{i=2}^{n} x_{i} y_{i}-\sum_{i=2}^{n} x_{i} v_{2, i} \\
& =\left(v_{2,1} / y_{1}\right)\left(\langle\mathbf{x}, \mathbf{y}\rangle-x_{1} y_{1}\right)-\left(\left\langle\mathbf{v}_{2}, \mathbf{x}\right\rangle-v_{2,1} x_{1}\right) \\
& =-\left\langle\mathbf{v}_{2}, \mathbf{x}\right\rangle .
\end{aligned}
$$


Challenge Phase: $\mathscr{A}$ provides two messages $m_{0}, m_{1}$ and a challenge vector $\widehat{\mathrm{x}}=\left(\widehat{x}_{1}, \ldots, \widehat{x}_{n}\right) . \mathscr{B}$ picks $\beta \stackrel{\mathrm{U}}{\longleftarrow}\{0,1\}$ and generates the challenge ciphertext $\widehat{\mathcal{C}}=\left(C_{0}, C_{1}, C_{2}, C_{3}, \widehat{\text { ctag }}\right)$ as follows.

$$
\begin{aligned}
& s, \mu \stackrel{\mathrm{U}}{\longleftarrow} \mathbb{Z}_{p}, \widehat{\mathrm{ctag}}=-\left\langle\mathbf{v}_{2}, \widehat{\mathbf{x}}\right\rangle \\
& C_{0}=m_{\beta} \cdot e\left(P_{1}, P_{2}\right)^{s \alpha} \cdot e\left(P_{1}, P_{2}\right)^{\mu \alpha_{1}}, \\
& C_{1}=s P_{1}+\mu P_{1}, C_{2}=s b P_{1}, C_{3}=s(\langle\mathbf{u}, \widehat{\mathbf{x}}\rangle+\operatorname{ctag} \cdot w) P_{1}+\mu\left(\left\langle\mathbf{v}_{1}, \mathbf{x}\right\rangle\right) P_{2} .
\end{aligned}
$$

The semi-functional component of $C_{3}$ is given by

$$
\begin{aligned}
\mu\left(\left\langle\mathbf{u}_{1}, \widehat{\mathbf{x}}\right\rangle+\widehat{\operatorname{ctag}} \cdot w_{1}\right) P_{2} & =\mu\left(\left\langle\mathbf{v}_{1}+w_{1} \mathbf{v}_{2}, \widehat{\mathbf{x}}\right\rangle-\widehat{\operatorname{ctag}} \cdot w_{1}\right) P_{2} \\
& =\mu\left(\left\langle\mathbf{v}_{1}, \widehat{\mathbf{x}}\right\rangle+w_{1}\left\langle\mathbf{v}_{2}, \widehat{\mathbf{x}}\right\rangle-\left\langle\mathbf{v}_{2}, \widehat{\mathbf{x}}\right\rangle w_{1}\right) P_{2} \\
& =\mu\left(\left\langle\mathbf{v}_{1}, \widehat{\mathbf{x}}\right\rangle\right) P_{2},
\end{aligned}
$$

and hence $C_{3}$ has the correct distribution. Another point to note is the following - $\mathscr{B}$ does not know an encoding of $w_{1}$ in $\mathbb{G}_{1}$. In order to generate a semifuntional ciphertext for a vector $\mathbf{x}$. the only choice for ctag is $-\left\langle\mathbf{v}_{2}, \mathbf{x}\right\rangle$. This helps in cancelling out the $w_{1} P_{1}$ component with the $w_{1}$-portion of the hash created using $\mathbf{u}_{1}=\mathbf{v}_{1}+w_{1} \mathbf{v}_{2}$.

If $\gamma=0, k$-th key is distributed as in $\mathrm{G}_{3, k-1}$; otherwise $\gamma \stackrel{\mathrm{U}}{\longleftarrow} \mathbb{Z}_{p}$ and $\mathcal{S} \mathcal{K}_{\mathbf{y}_{k}}$ is distributed as a semi-functional key. Furthermore, if $\mathscr{B}$ tries to create a ciphertext for a vector $\mathbf{x}$ that is orthogonal to $\mathbf{y}_{k}$, it can only do so by setting ctag $=-\left\langle\mathbf{v}_{2}, \mathbf{x}\right\rangle=$ ktag. In this case decryption would fail unconditionally and provide no information to $\mathscr{B}$ about the key for $\mathbf{y}_{k}$. What remains is to show that all the information provided to $\mathscr{A}$ are properly distributed. The public parameters are distributed as in the real scheme. Randomisers for the keys and ciphertexts including their semi-functional components have the correct distribution. We only need to show that all the $n$ tags - $\widehat{\text { ctag }}$ of the challenge ciphertext and $\operatorname{ktag}_{2}, \ldots, \operatorname{ktag}_{n}$ for the $k$-th secret key - generated using $\mathbf{v}_{2}$, are randomly distributed in $\mathbb{Z}_{p}$. The tags are defined in the reduction as follows.

$$
\left(\begin{array}{c}
\widehat{\operatorname{ctag}} \\
\operatorname{ktag}_{2} \\
\vdots \\
\operatorname{ktag}_{n}
\end{array}\right)=\left(\begin{array}{ccccc}
-\widehat{x}_{1} & -\widehat{x}_{2} & -\widehat{x}_{3} & \cdots & -\widehat{x}_{n} \\
y_{2} / y_{1} & -1 & 0 & \cdots & 0 \\
y_{3} / y_{1} & 0 & -1 & \cdots & 0 \\
\vdots & \vdots & \vdots & \ddots & \vdots \\
y_{n} / y_{1} & 0 & 0 & \cdots & -1
\end{array}\right)\left(\begin{array}{c}
v_{2,1} \\
v_{2,2} \\
\vdots \\
v_{2, n}
\end{array}\right)
$$

All information provided to the adversary, including public parameters are computed independent of $\mathbf{v}_{2}$. Since $\mathbf{v}_{2}$ is chosen uniformly from $\mathbb{Z}_{p}^{n}$, the tags are uniformly distributed in the adversary's view if the determinant of the matrix above is non-zero. It is easy to observe that determinant is $(-1)^{n}\left\langle\widehat{\mathbf{x}}, \mathbf{y}_{k}\right\rangle / y_{1}$ which is non-zero because all of $\mathscr{A}$ 's queries are such that $\left\langle\widehat{\mathbf{x}}, \mathbf{y}_{k}\right\rangle \neq 0$. Therefore, the simulation is perfect.

\section{B Proof of Theorem 2}

We first describe the semi-functional ciphertext and key generation algorithms required for the proof. 
$I \mathcal{P E}_{2}$. SFEncrypt $(\mathcal{P} \mathcal{P}, \mathcal{M S K}, m, \mathbf{x})$ : Generate

$\left.\left(C_{0}, C_{1}, C_{2},\left(C_{3, i}, \operatorname{ctag}_{i}\right)_{i=2}^{n}\right)\right) \stackrel{\mathrm{R}}{\longleftarrow} I \mathcal{P E} \mathcal{E}_{2}$. Encrypt $(\mathcal{P} \mathcal{P}, m, \mathbf{x})$. Choose $\mu \stackrel{\mathrm{U}}{\longleftarrow} \mathbb{Z}_{p}$ and generate the semi-functional ciphertext components as follows.

$$
\begin{aligned}
& C_{0} \longleftarrow C_{0} \cdot e\left(P_{1}, P_{2}\right)^{\mu \alpha_{1}}, \\
& C_{1} \longleftarrow C_{1}+\mu P_{1}, \\
& C_{3, i} \longleftarrow C_{3, i}+\mu\left(-\frac{x_{i}}{x_{1}} u_{1,1}+u_{1, i}+\operatorname{ctag}_{i} w_{1}\right) P_{1} \text { for } i=2, \ldots, n .
\end{aligned}
$$

Return $\left.\mathcal{C}=\left(C_{0}, C_{1}, C_{2}, C_{3},\left(C_{3, i}, \operatorname{ctag}_{i}\right)_{i=2}^{n}\right)\right)$ as the resulting semi-functional ciphertext.

$I \mathcal{P E}_{2}$.SFKeyGen $(\mathcal{P} \mathcal{P}, \mathcal{M S K}, \mathbf{y})$ : Let $\mathcal{S K}_{\mathbf{y}}^{\prime}=\left(K_{1}, K_{2}, K_{3}, K_{4}, K_{5}\right)$ be obtained by running $I P E_{2}$. KeyGen $(\mathcal{M S K}, \mathbf{y})$. Pick $\gamma, \pi \stackrel{\mathrm{U}}{\longleftarrow} \mathbb{Z}_{p}$ and modify the components of $\mathcal{S K}_{\mathbf{y}}^{\prime}$ as follows:

$$
\begin{aligned}
& K_{2} \longleftarrow K_{2}+\gamma \pi P_{2}, K_{3} \longleftarrow K_{3}-\frac{\gamma \pi}{b} P_{2}, \\
& K_{4} \longleftarrow K_{4}+\gamma P_{2}, K_{5} \longleftarrow K_{5}-\frac{\gamma}{b} P_{2} .
\end{aligned}
$$

The semi-functional key given by $\mathcal{S K}_{\mathbf{y}}^{\prime}=\left(K_{1}, K_{2}, K_{3}, K_{4}, K_{5}\right)$ is returned as output.

When a semi-functional ciphertext is decrypted with a semi-functional key, the message is blinded by a factor $e\left(P_{1}, P_{2}\right)^{\mu \gamma(\pi+\mathrm{ctag})}$ as a result of pairing $C_{1}$ with $K_{2}+\operatorname{ctag} K_{4}$, the rest of the semi-functional terms being canceled. If the relation $\pi=-$ ctag holds, then decryption succeeds. In this case, we call the ciphertext and key nominally semi-functional.

The proof is a hybrid argument over a sequence of $q+6$ games $\mathrm{G}_{0}, \mathrm{G}_{1}, \mathrm{G}_{2}$, $\mathrm{G}_{3,0}, \ldots, \mathrm{G}_{3, q}, \mathrm{G}_{4}, \mathrm{G}_{5}$ where $\mathrm{G}_{0}$ is the real ind-wah-cpa attack game defined in Section 2.3. Let $X_{\square}$ denote the event that the adversary $\mathscr{A}$ wins (i.e., $\beta=\beta^{\prime}$ ) in $\mathrm{G}_{\square}$. The rest of the games are described below.

Game $G_{1}$ : Defined similar to $G_{0}$ except for a modification to the Encrypt algorithm. Elements from the master secret key $\mathcal{M S K}$ are used instead of the public parameters to generate the challenge ciphertext.

$$
\begin{aligned}
& \left(\widehat{\operatorname{ctag}}_{i}\right)_{i=2}^{n}, s \stackrel{\mathrm{U}}{\mathbb{Z}_{p}}, \\
& C_{1}=s P_{1}, C_{2}=s b P_{1}, \\
& C_{0}=m_{\beta} \cdot\left(C_{1}, P_{2}\right)^{\alpha_{1}} e\left(C_{2}, P_{2}\right)^{\alpha_{2}} \\
& \text { for } i=2, \ldots, n, \\
& C_{3, i}=\left(-\frac{\widehat{x}_{\beta, i}}{\widehat{x}_{\beta, 1}} u_{1,1}+u_{1, i}+\operatorname{ctag}_{i} w_{1}\right) C_{1}+\left(-\frac{\widehat{x}_{\beta, i}}{\widehat{x}_{\beta, 1}} u_{2,1}+u_{2, i}+\operatorname{ctag}_{i} w_{2}\right) C_{2} .
\end{aligned}
$$

It is not hard to see that $C_{0}$ and $C_{3}$ are well-formed. The change we have made is only conceptual and hence $G_{1}$ is identical to $G_{0}$. We have

$$
\operatorname{Pr}\left[X_{0}\right]=\operatorname{Pr}\left[X_{1}\right]
$$


Game $\mathrm{G}_{2}$ : The challenge ciphertext generation in $\mathrm{G}_{1}$ is changed to generate $C_{1}$ as $C_{1} \stackrel{\mathrm{U}}{\longleftarrow} \mathbb{G}_{1}$. We can show that an adversary capable of detecting this change can be used to solve DDH1. In other words, we have the following lemma.

Lemma 3. $\left|\operatorname{Pr}\left[X_{1}\right]-\operatorname{Pr}\left[X_{2}\right]\right| \leq \varepsilon_{\mathrm{DDH} 1}$.

It is a rather straightforward reduction wherein we embed the DDH1 challenge in $C_{1}$. The proof is similar to that of Lemma 1 and hence we skip it.

Game $G_{3,0}$ : We arrive at $G_{3,0}$ by modifying the setup, key generation and encryption procedures in $G_{2}$ as follows.

Setup: The public parameters are generated as follows. Choose $P_{1} \stackrel{U}{\longleftarrow} \mathbb{G}_{1}^{\times}$, $P_{2} \stackrel{\mathrm{U}}{\longleftarrow} \mathbb{G}_{2}^{\times}, \alpha, \alpha_{1}, w, w_{1} \stackrel{\mathrm{U}}{\longleftarrow} \mathbb{Z}_{p}, b \stackrel{\mathrm{U}}{\longleftarrow} \mathbb{Z}_{p}^{\times}, \mathbf{u}, \mathbf{v}_{1}, \mathbf{v}_{2} \stackrel{\mathrm{U}}{\longleftarrow} \mathbb{Z}_{p}^{n}$ and compute $\mathbf{u}_{1}=\mathbf{v}_{1}+w_{1} \mathbf{v}_{2}, g_{T}=e\left(P_{1}, P_{2}\right)^{\alpha}$. The algorithm implicitly sets $\mathbf{u}_{2}=b^{-1}(\mathbf{u}-$ $\left.\mathbf{u}_{1}\right), w_{2}=b^{-1}\left(w-w_{1}\right)$ and $\alpha_{2}=b^{-1}\left(\alpha-\alpha_{1}\right)$. The parameters are given by $\mathcal{P P}=\left(P_{1}, b P_{1}, \mathbf{u} P_{1}, w P_{1}, g_{T}\right)$.

Key Generation: On input a vector $\mathbf{y}$, the KeyGen algorithm is modified to create the components of $\mathcal{S} \mathcal{K}_{\mathbf{y}}$ as shown below.

$$
\begin{aligned}
& r \stackrel{\mathrm{U}}{\breve{Z}} \mathbb{Z}_{p}, \\
& K_{1}=r P_{2}, \\
& K_{2}=\left(\alpha_{1}+r\left\langle\mathbf{y}, \mathbf{v}_{1}\right\rangle\right) P_{2}+\left\langle\mathbf{y}, \mathbf{v}_{2}\right\rangle K_{4}, K_{3}=b^{-1}\left((\alpha+r\langle\mathbf{y}, \mathbf{u}\rangle) P_{2}-K_{2}\right) \\
& K_{4}=r w_{1} P_{2}, K_{5}=b^{-1}\left(r w P_{2}-K_{4}\right) .
\end{aligned}
$$

Encryption: The Encrypt algorithm is modified to use public parameters instead of elements from the master secret (as in the construction). The challenge ciphertext is generated by a call to the SFEncrypt algorithm. Essentially, the components of $\widehat{\mathcal{C}}$ are generated as follows.

$$
\begin{aligned}
& \left(\widehat{\operatorname{ctag}}_{i}\right)_{i=2}^{n}, s, \mu \stackrel{\mathrm{U}}{\mathbb{Z}_{p},} \\
& C_{0}=m_{\beta} \cdot\left(g_{T}\right)^{s} e\left(P_{1}, P_{2}\right)^{\mu \alpha_{1}}, \\
& C_{1}=s P_{1}+\mu P_{1}, C_{2}=s b P_{1}, \\
& \text { for } i=2, \ldots, n, \\
& C_{3, i}=s\left(-\widehat{x}_{\beta, i} \widehat{x}_{\beta, 1} u_{1}+u_{i}+\widehat{\operatorname{ctag}}_{i} w\right) P_{1}+\mu\left(-\widehat{x}_{\beta, i} \widehat{x}_{\beta, 1} u_{1,1}+u_{1, i}+\widehat{\operatorname{ctag}}_{i} w_{1}\right) P_{1} .
\end{aligned}
$$

The modifications are purely conceptual and so game $G_{3,0}$ is identical to $G_{2}$. We have

$$
\operatorname{Pr}\left[X_{2}\right]=\operatorname{Pr}\left[X_{3,0}\right]
$$

Game $\mathrm{G}_{3, k}$ (for $k=1, \ldots, q$ ): Let $\mathbf{y}_{1}, \ldots, \mathbf{y}_{q}$ be the vectors queried by $\mathscr{A}$. This game is identical to $\mathrm{G}_{3, k-1}$ except for the following two modifications. The $(k-1)$-st key is generated according to the SFKeyGen algorithm and $\mathcal{S} \mathcal{K}_{\mathbf{y}_{k}}$ for the $k$-th vector $\mathbf{y}_{k}$ is generated differently - component $K_{2}$ of $\mathcal{S K}_{\mathbf{y}_{k}}$ is now chosen uniformly at random from $\mathbb{G}_{2}$. The first change is purely conceptual and hence does not affect the adversary's behaviour in any way. We show that if the adversary can detect the second modification, then the DDH2 problem can be solved. We have the following lemma.

Lemma 4. $\left|\operatorname{Pr}\left[X_{3, k-1}\right]-\operatorname{Pr}\left[X_{3, k}\right]\right| \leq \varepsilon_{\mathrm{DDH} 2}$ for all $k \in[1, q]$. 
$\mathrm{G}_{4}$ : Proceeds identical to $\mathrm{G}_{3, q}$ but for one difference - the secret key corresponding to $\mathbf{y}_{q}$ is computed using the SFKeyGen algorithm. Since this is only a conceptual change, we have

$$
\operatorname{Pr}\left[X_{3, q}\right]=\operatorname{Pr}\left[X_{4}\right]
$$

Game $G_{5}$ : We arrive at this game from $G_{4}$ by modifying the setup and semifunctional key generation algorithms.

Setup: The public parameters are generated as follows. Choose $P_{1} \stackrel{\mathrm{U}}{\longleftarrow} \mathbb{G}_{1}^{\times}$, $P_{2} \stackrel{\mathrm{U}}{\longleftarrow} \mathbb{G}_{2}^{\times}, \alpha, \alpha_{1}, w, w_{1} \stackrel{\mathrm{U}}{\longleftarrow} \mathbb{Z}_{p}, b \stackrel{\mathrm{U}}{\longleftarrow} \mathbb{Z}_{p}^{\times}, \mathbf{u}, \mathbf{u}_{1} \stackrel{\mathrm{U}}{\longleftarrow} \mathbb{Z}_{p}^{n}$ and compute $g_{T}=$ $e\left(P_{1}, P_{2}\right)^{\alpha}$. The algorithm implicitly sets $\mathbf{u}_{2}=b^{-1}\left(\mathbf{u}-\mathbf{u}_{1}\right), w_{2}=b^{-1}\left(w-w_{1}\right)$ and $\alpha_{2}=b^{-1}\left(\alpha-\alpha_{1}\right)$ and outputs $\mathcal{P} \mathcal{P}=\left(P_{1}, b P_{1}, \mathbf{u} P_{1}, w P_{1}, g_{T}\right)$.

Key Generation: Let $\mathbf{y}$ be the input to SFKeyGen.

$$
\begin{aligned}
& r, \pi^{\prime}, \gamma^{\prime} \stackrel{\mathrm{U}}{\longleftarrow} \mathbb{Z}_{p}, \\
& K_{1}=r P_{2}, \\
& K_{2}=\pi^{\prime} P_{2}, K_{3}=b^{-1}\left((\alpha+r\langle\mathbf{y}, \mathbf{u}\rangle) P_{2}-K_{2}\right), \\
& K_{4}=\gamma^{\prime} P_{2}, K_{5}=b^{-1}\left((\alpha+r w) P_{2}-K_{4}\right)
\end{aligned}
$$

The rest of the secret key components are generated as in $\mathrm{G}_{3,0}$.

The reduction implicitly sets $\gamma^{\prime}=\alpha_{1}+r\left\langle\mathbf{y}, \mathbf{u}_{1}\right\rangle P_{2}+\pi \gamma$, thus fixing $\pi$. The rest of the components are consistent with $K_{2}$. If $\gamma \neq 0, \pi$ is uniformly distributed in $\mathbb{Z}_{p}$ by the choice of $\gamma^{\prime}$. Note that even if $\gamma=0, \pi$ can take any value from $\mathbb{Z}_{p}$ uniformly at random. Hence the keys are correctly distributed.

Since the change is only conceptual, we have,

$$
\left|\operatorname{Pr}\left[X_{4}\right]-\operatorname{Pr}\left[X_{5}\right]\right| \leq(1 / p) .
$$

Now consider the challenge ciphertext. All keys are computed independent of $\alpha_{1}, \mathbf{u}_{1}, w_{1}$ and so are the public parameters. As a result, in the adversary's view, $e\left(P_{1}, P_{2}\right)^{\mu \alpha_{1}}$ is randomly distributed in $\mathbb{G}_{T}$ as long as $\mu \neq 0$ which happens with probability at most $1 / p$. The semi-functional components of $C_{3, i}, i \in[2, n]$ are determined by the following matrix-vector product.

$$
\left(\begin{array}{cccccc}
\widehat{x}_{\beta, 2} / \widehat{x}_{\beta, 1} & 1 & 0 & \cdots & 0 \\
\widehat{x}_{\beta, 3} / \widehat{x}_{\beta, 1} & 0 & 1 & \cdots & 0 \\
\vdots & \vdots & \vdots & \ddots & \vdots \\
\widehat{x}_{\beta, n} / \widehat{x}_{\beta, 1} & 0 & 0 & \cdots & 1
\end{array}\right)\left(\begin{array}{c}
u_{1,1} \\
u_{1,2} \\
\vdots \\
u_{1, n}
\end{array}\right) .
$$

The rank of the matrix is $n-1$ and hence the semi-functional components are uniformly distributed in the adversary's view (given that $\mathbf{u}_{1} \stackrel{U}{\longleftarrow} \mathbb{Z}_{p}^{n}$ and $\mu \neq 0$ ).

Based on the discussion above we can say that in game $\mathrm{G}_{5}$, all components involving the message $m_{\beta}$ and the attribute vector $\widehat{x x}_{\beta}$ are randomly distributed. Hence the bit $\beta$ is information theoretically hidden from the adversary. The 
probability that $\mathscr{A}$ wins in $G_{5}$ is exactly $1 / 2$. Therefore, from Lemmas 3,4 and equations (5), (6), (7) and (8), we have

$$
\begin{aligned}
\varepsilon= & \left|\operatorname{Pr}\left[X_{0}\right]-\frac{1}{2}\right| \\
= & \left|\operatorname{Pr}\left[X_{0}\right]-\operatorname{Pr}\left[X_{5}\right]\right| \\
\leq & \left|\operatorname{Pr}\left[X_{0}\right]-\operatorname{Pr}\left[X_{1}\right]\right|+\left|\operatorname{Pr}\left[X_{1}\right]-\operatorname{Pr}\left[X_{2}\right]\right|+\left|\operatorname{Pr}\left[X_{2}\right]-\operatorname{Pr}\left[X_{3}, 0\right]\right| \\
& +\left(\sum_{k=1}^{q}\left|\operatorname{Pr}\left[X_{3, k-1}\right]-\operatorname{Pr}\left[X_{3, k}\right]\right|\right)+\left|\operatorname{Pr}\left[X_{3, q}\right]-\operatorname{Pr}\left[X_{4}\right]\right|+\left|\operatorname{Pr}\left[X_{4}\right]-\operatorname{Pr}\left[X_{5}\right]\right| \\
\leq & \varepsilon_{\mathrm{DDH} 1}+q \cdot \varepsilon_{\mathrm{DDH} 2}+(1 / p)
\end{aligned}
$$

Proof (of Lemma 4). If the attacker can distinguish between the two games $\mathrm{G}_{3, k-1}$ and $\mathrm{G}_{3, k}$, then we show how to build an algorithm $\mathscr{B}$ that solves the DDH2 problem. Let $\left(\mathcal{G}, P_{2}, r P_{2}, w_{1} P_{2},\left(r w_{1}+\gamma\right) P_{2}\right)$ be an instance of DDH2 provided to $\mathscr{B}$ whose task is to determine whether $\gamma=0$ or $\gamma \stackrel{\mathrm{U}}{\longleftarrow} \mathbb{Z}_{p}$. The different phases of the game are simulated as follows.

Setup: Choose $P_{1} \stackrel{\mathrm{U}}{\longleftarrow} \mathbb{G}_{1}^{\times}, \alpha, \alpha_{1}, w, w_{1} \stackrel{\mathrm{U}}{\longleftarrow} \mathbb{Z}_{p}, b \stackrel{\mathrm{U}}{\longleftarrow} \mathbb{Z}_{p}^{\times}, \mathbf{u}, \mathbf{v}_{1}, \mathbf{v}_{2} \stackrel{\mathrm{U}}{\longleftarrow} \mathbb{Z}_{p}^{n}$ and compute $\mathbf{u}_{1} P_{2}=\mathbf{v}_{1} P_{2}+\mathbf{v}_{2}\left(w_{1} P_{2}\right), g_{T}=e\left(P_{1}, P_{2}\right)^{\alpha}$. The algorithm implicitly sets $\mathbf{u}_{2}=b^{-1}\left(\mathbf{u}-\mathbf{u}_{1}\right), w_{2}=b^{-1}\left(w-w_{1}\right)$ and $\alpha_{2}=b^{-1}\left(\alpha-\alpha_{1}\right)$. The parameters are given by $\mathcal{P} \mathcal{P}=\left(P_{1}, b P_{1}, \mathbf{u} P_{1}, w P_{1}, g_{T}\right)$. Note that $\mathcal{P} \mathcal{P}$ can be generated without the knowledge of $\mathbf{u}_{1}$ or $w_{1}$.

Key Generation: Denote by $\mathbf{y}_{1}, \ldots, \mathbf{y}_{q}$ the vectors queried by $\mathscr{A}$. Normal keys can be generated as in $\mathrm{G}_{3,0}$. For $j=1, \ldots, q$, key $\mathcal{S} \mathcal{K}_{\mathbf{y}_{j}}$ is generated as follows.

Case $j<k$ : Use SFKeyGen to generate $\mathcal{S K}_{\mathbf{y}_{j}}$. This is possible since $b$ is known to $\mathscr{B}$.

Case $j=k$ : Create the key $\mathcal{S K}_{\mathbf{y}_{k}}=\left(K_{1}, K_{2}, K_{3}, K_{4}, K_{5}\right)$ as follows. Let $\mathbf{y}_{k}=$ $\left(y_{1}, \ldots, y_{n}\right)$.

Pick $r \stackrel{\mathrm{U}}{\longleftarrow} \mathbb{Z}_{p}$ and set,

$K_{1}=r P_{2}$,

$K_{2}=\left(\alpha_{1}+r\left\langle\mathbf{y}, \mathbf{v}_{1}\right\rangle\right) P_{2}+\left\langle\mathbf{y}, \mathbf{v}_{2}\right\rangle K_{4}, K_{3}=b^{-1}\left((\alpha+r\langle\mathbf{y}, \mathbf{u}\rangle) P_{2}-K_{2}\right)$

$K_{4}=\left(r w_{1}+\gamma\right) P_{2}, K_{5}=b^{-1}\left(r w P_{2}-K_{4}\right)$.

Components of $\mathcal{S} \mathcal{K}_{\mathbf{y}_{k}}$ are generated exactly as in game $\mathrm{G}_{3,0}$ except that the DDH2 challenge is embedded in $K_{4}$.

Case $j>k$ : $\mathscr{B}$ computes a normal key according to the KeyGen algorithm.

Challenge Phase: $\mathscr{A}$ provides two messages $m_{0}, m_{1}$ and two vectors $\widehat{\mathbf{x}}_{0}=$ $\left(\widehat{x}_{0,1}, \ldots, \widehat{x}_{0, n}\right), \widehat{\mathbf{x}}_{1}=\left(\widehat{x}_{1,1}, \ldots, \widehat{x}_{1, n}\right) . \mathscr{B}$ picks $\beta \stackrel{U}{\longleftarrow}\{0,1\}$ and generates the challenge ciphertext $\widehat{\mathcal{C}}=\left(C_{0}, C_{1}, C_{2},\left(C_{3, i}, \widehat{\operatorname{ctag}}_{i}\right)_{i=2}^{n}\right)$ as follows.

Pick $s, \mu \stackrel{\mathrm{U}}{\longleftarrow} \mathbb{Z}_{p}$ and set, $\widehat{\operatorname{ctag}}_{i}=\frac{\widehat{x}_{\beta, i}}{\widehat{x}_{\beta, 1}} v_{2,1}-v_{2, i}$, 


$$
\begin{aligned}
& C_{0}=m_{\beta} \cdot\left(g_{T}\right)^{s} e\left(P_{1}, P_{2}\right)^{\mu \alpha_{1}}, \\
& C_{1}=s P_{1}+\mu P_{1}, C_{2}=s b P_{1}, \\
& \text { for } i=2, \ldots, n, \\
& C_{3, i}=s\left(-\frac{\widehat{x}_{\beta, i}}{\widehat{x}_{\beta, 1}} u_{1}+u_{i}+\widehat{\operatorname{ctag}}_{i} w\right) P_{1}+\mu\left(-\widehat{x}_{\beta, i} v_{1,1}+v_{1, i}\right) P_{1} .
\end{aligned}
$$

The choice of tags above enables us to create the semi-functional component without the knowledge of $w_{1}$. Essentially we cancel out the $\mathbf{v}_{2}$ component of hash (computed using $\mathbf{u}_{1}=\mathbf{v}_{1}+w_{1} \mathbf{v}_{2}$ ) by choosing the corresponding tag for $w_{1}$-component appropriately. We stress that this is the only way to create a semi-functional ciphertext.

If $\gamma=0, k$-th key is distributed as in $\mathrm{G}_{3, k-1}$; otherwise $\gamma \stackrel{\mathrm{U}}{\longleftarrow} \mathbb{Z}_{p}$ and $\mathcal{S K}_{\mathbf{y}_{k}}$ is distributed as a semi-functional key with randomiser $\gamma$ coming from the DDH2 challenge and $\pi=\left\langle\mathbf{x}, \mathbf{v}_{2}\right\rangle$. Now, suppose that $\mathscr{B}$ tries to create a ciphertext for a vector $\mathbf{x}$ that is orthogonal to $\mathbf{y}_{k}$, it can only do so by setting $\operatorname{ctag}_{i}=\frac{x_{i}}{x_{1}} v_{2,1}-v_{2, i}$. This implies that

$$
\begin{aligned}
\operatorname{ctag} & =\sum_{i=2}^{n} y_{i} \operatorname{ctag}_{i} \\
& =\sum_{i=2}^{n} y_{i}\left(\left(x_{i} / x_{1}\right) v_{2,1}-v_{2, i}\right) \\
& =\left(v_{2,1} / x_{1}\right) \sum_{i=2}^{n} x_{i} y_{i}-\sum_{i=2}^{n} y_{i} v_{2, i} \\
& =\left(v_{2,1} / x_{1}\right)\left(\langle\mathbf{x}, \mathbf{y}\rangle-x_{1} y_{1}\right)-\left(\left\langle\mathbf{v}_{2}, \mathbf{y}\right\rangle-v_{2,1} y_{1}\right) \\
& =-\left\langle\mathbf{v}_{2}, \mathbf{y}\right\rangle,
\end{aligned}
$$

which is equal to $-\pi$, which is precisely the requirement for the ciphertext and key to be nominally semi-functional. Decryption succeeds and $\mathscr{B}$ obtains no information about whether or not $\mathcal{S} \mathcal{K}_{\mathbf{y}_{k}}$ is semi-functional.

We now show that the view of the adversary is perfectly simulated by $\mathscr{B}$. The public parameters are distributed as in the real scheme. Randomisers for the keys and ciphertexts including their semi-functional components have the correct distribution. We only need to show that all the $n-1 \operatorname{tags,}\left(\widehat{\operatorname{ctag}}_{i}\right)_{i=2}^{n}$ of the challenge ciphertext and $\pi$ for the $k$-th secret key - generated using $\mathbf{v}_{2}$, are randomly distributed in $\mathbb{Z}_{p}$.

$$
\left(\begin{array}{c}
\frac{\pi}{\operatorname{ctag}_{2}} \\
\vdots \\
\widehat{\operatorname{ctag}}_{n}
\end{array}\right)=\left(\begin{array}{ccccc}
y_{1} & y_{2} & y_{3} & \cdots & y_{n} \\
\widehat{x}_{\beta, 2} / \widehat{x}_{\beta, 1} & -1 & 0 & \cdots & 0 \\
\widehat{x}_{\beta, 3} / \widehat{x}_{\beta, 1} & 0 & -1 & \cdots & 0 \\
\vdots & \vdots & \vdots & \ddots & \vdots \\
\widehat{x}_{\beta, n} / \widehat{x}_{\beta, 1} & 0 & 0 & \cdots & -1
\end{array}\right)\left(\begin{array}{c}
v_{2,1} \\
v_{2,2} \\
\vdots \\
v_{2, n}
\end{array}\right)
$$

From the above equation, one can say that the tags and $\pi$ have uniform and independent distributions over $\mathbb{Z}_{p}$ in $\mathscr{A}$ 's view iff 
- $\mathbf{v}_{2}$ is information theoretically hidden in $\mathscr{A}$ 's view and uniformly distributed in $\mathbb{Z}_{p}^{n}$,

- the matrix is invertible.

The first condition is naturally satisfied as no encodings of $\mathbf{v}_{2}$ in either $\mathbb{G}_{1}$ or $\mathbb{G}_{2}$ are revealed in the public parameters. The second condition also holds since the determinant of the matrix is $(-1)^{n}\left\langle\widehat{\mathbf{x}}, \mathbf{y}_{k}\right\rangle / \widehat{x}_{\beta, 1}$ which is clearly non-zero due to the restriction on $\mathscr{A}$ 's queries requiring $\left\langle\widehat{\mathbf{x}}, \mathbf{y}_{k}\right\rangle \neq 0$. Therefore, the simulation is perfect.

\section{Application to Identity-Based Broadcast Encryption}

We explain how to derive an identity-based encryption scheme (IBBE) that has sub-linear sized ciphertexts and keys. In an IBBE scheme, each user is associated with an identity id $\in \mathcal{I}$ ( $\mathcal{I}$ denotes the domain of identities). There is a central authority called the private key generator $(\mathrm{PKG})$ that generates and distributes keys for all users securely. The secret key corresponding to a user with identity id is denoted $\mathcal{S} \mathcal{K}_{\text {id }}$. A sender can broadcast an encrypted message (as in IPE, the parameters of the PKG are used for encryption) to all users of the system. The message however is only intended for a subset $S=\left\{\mathrm{id}_{1}, \ldots, \mathrm{id}_{\ell}\right\}$ of users that we refer to as privileged. Naturally, this requires the ciphertext to be constructed in a manner that allows only a legitimate/privileged user to decrypt. In other words, only a user with identity id with id $\in S$ can decrypt the ciphertext using $\mathcal{S} \mathcal{K}_{\text {id }}$.

An IBBE can be expressed as a special case of inner product encryption. We only provide the top-level idea and skip the details. As our constructions are based on bilinear groups of prime order $p$, let the domain of attributes and identities to be $\mathbb{Z}_{p}$. We now show that an IPE scheme supporting inner products over $\mathbb{Z}_{p}^{n+1}$ can be specialised to an IBBE scheme where the number of intended recipients of a broadcast is upper bounded by $n$. Consider a particular set $S=$ $\left\{\operatorname{id}_{1}, \ldots, \mathrm{id}_{\ell}\right\}$ with $\ell \leq n$. Define the polynomial $p_{S}(z)=\prod_{i=1}^{\ell}\left(z-\mathrm{id}_{i}\right) \in \mathbb{Z}_{p}[z]$ and compute $\mathbf{x}=\left(x_{0}, \ldots, x_{n}\right)$, the vector of coefficients of $1, z, z^{2}, \ldots, z^{n}$ in $p_{S}(z)$. Since the degree of the polynomial is $\ell, x_{j}=0$ for all $j>\ell$. The ciphertext is encrypted to the attribute vector $\mathbf{x}$ as in the Encrypt alrogithm of the IPE. The secret key for an identity id is constructed as follows: let $\mathbf{y}=\left(1, \mathrm{id}_{\mathrm{id}}{ }^{2}, \ldots, \mathrm{id}^{n}\right)$; create a key for the vector $\mathbf{y}$ using the IPE KeyGen algorithm. If id $\in S$, then clearly id is a root of $p_{S}(z)$ and hence $\langle\mathbf{y}, \mathbf{x}\rangle=x_{0}+\mathrm{id} x_{1}+\mathrm{id}^{2} x_{2}+\cdots+\mathrm{id}^{n} x_{n}=0$. The converse also holds.

Following the method outlined above, we can derive a constant-size ciphertext IBBE from $I \mathscr{P E} \mathcal{E}_{1}$ with ciphertext size being $3\left|\mathbb{G}_{1}\right|+\left|\mathbb{Z}_{p}\right|+\left|\mathbb{G}_{T}\right|$ (recall that $|\mathbb{G}|$ is the size of representation of an element from $\mathbb{G}$ ). Size of a key would be $(2 n+3)\left|\mathbb{G}_{2}\right|+n\left|\mathbb{Z}_{p}\right|$. Similarly, the scheme IPE $\mathcal{E}_{2}$ leads to an IBBE with constantsize keys (of size $5\left|\mathbb{G}_{2}\right|$ ) and ciphertext of size $(n+2)\left|\mathbb{G}_{1}\right|+n\left|\mathbb{Z}_{p}\right|+\left|\mathbb{G}_{T}\right|$. In addition this scheme is anonymous i.e., decryption can be performed without knowing the set for which the ciphertext was encrypted to. Furthermore, unlike 
many anonymous broadcast encryption schemes, decryption time is constant (independent of $\ell$ and $n$ ).

In the IBBE obtained from $I \mathcal{P E} \mathcal{E}_{1}$, the linear dependence of key size on $n$ is rather impractical in certain scenarios, for instance, when the user keys are actually stored in smartcards. On the other hand, ciphertext size also contributes to the communication overhead and longer ciphertexts consume larger bandwidth. So, IPE $\mathcal{E}_{2}$ ciphertexts indeed eat up larger bandwidth. Also, these ciphertexts grow linearly in $n$, unlike the construction in [RS14a] where the ciphertext size varies with $\ell(\leq n)$. In order to strike a balance between the two, we propose sim-

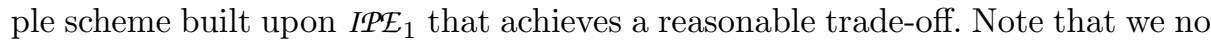
longer aim for ciphertext anonymity. A high-level overview of this construction is provided below.

As earlier, let $n$ denote the maximum number of privileged recipients of a broadcast and assume for simplicity that $n=n_{1} n_{2}$. Setup IPE 1 for inner products over $\mathbb{Z}_{p}^{n_{1}+1}$. The encryption algorithm partitions the input set $S=$ $\left\{\mathrm{id}_{1}, \ldots\right.$, id $\left._{\ell}\right\}$ into $\ell_{2}\left(\leq n_{2}\right)$ subsets $S_{1}, \ldots, S_{\ell_{2}}$ with $\left|S_{1}\right|=\left|S_{2}\right|=\cdots=\left|S_{\ell_{2}-1}\right|=$ $n_{1}$ and $\left|S_{\ell_{2}}\right|=\ell_{1}\left(\leq n_{1}\right)$. Then perform separate $I P E_{2}$-encryptions of the message for each of the sets. Secret keys are generated as in $I \mathcal{P E} \mathcal{E}_{2}$. Let $p_{S_{i}}(z)$ denote the polynomial corresponding to set $S_{i}$ (for $i \in\left[1, \ell_{2}\right]$ ) and $\mathbf{x}_{i}$ the corresponding coefficient vector. Let $\mathbf{y}$ denote the vector associated to the secret key for id. Now, any id $\in S$ is present exactly in one of the subsets, say $S_{j}$ implying that $\left\langle\mathbf{x}_{j}, \mathbf{y}\right\rangle=$ 0 and $\left\langle\mathbf{x}_{i}, \mathbf{y}\right\rangle \neq 0$ for $i \in\left[1, \ell_{2}\right] \backslash\{j\}$. The decryption algorithm can simply choose the subset to which id belongs and decrypt the $I P \mathcal{E}_{2}$-ciphertext corresponding to that set. On evaluating the efficiency of this scheme, we find that ciphertexts are of size $\ell_{2}\left(3\left|\mathbb{G}_{1}\right|+\left|\mathbb{Z}_{p}\right|+\left|\mathbb{G}_{T}\right|\right)$ and the key size is $\left(2 n_{1}+3\right)\left|\mathbb{G}_{1}\right|+n_{1}\left|\mathbb{Z}_{p}\right|$. This provides a nice trade-off among the public parameters, ciphertext and key sizes. Choosing $n_{1}$ and $n_{2}$ to be about sqrtn, we obtain an IBBE scheme with public parameters, ciphertexts and keys, all with $O(\sqrt{n})$-size, which is sublinear in $n$. 